DRAFT V1

Preprint typeset using $\mathrm{LATEX}_{\mathrm{E}}$ style emulateapj v. 12/16/11

\title{
EFFICIENCY OF SYNCHROTRON RADIATION FROM ROTATION-POWERED PULSARS
}

\author{
Shota Kisaka ${ }^{1,2}$ And Shuta J. TAnaka ${ }^{3}$ \\ draft $v 1$
}

\begin{abstract}
Synchrotron radiation is widely considered as the origin of the pulsed non-thermal emissions from rotation-powered pulsars in optical and X-ray bands. In this paper, we study the synchrotron radiation emitted by the created electron and positron pairs in the pulsar magnetosphere to constrain on the energy conversion efficiency from the Poynting flux to the particle energy flux. We model two pair creation processes, two-photon collision which efficiently works in young $\gamma$-ray pulsars $\left(\lesssim 10^{6} \mathrm{yr}\right)$, and magnetic pair creation which is the dominant process to supply pairs in old pulsars $\left(\gtrsim 10^{6} \mathrm{yr}\right)$. Using the analytical model, we derive the maximum synchrotron luminosity as a function of the energy conversion efficiency. From the comparison with observations, we find that the energy conversion efficiency to the accelerated particles should be an order of unity in the magnetosphere, even though we make a number of the optimistic assumptions to enlarge the synchrotron luminosity. In order to explain the luminosity of the non-thermal X-ray/optical emission from pulsars with low spin-down luminosity $L_{\mathrm{sd}} \lesssim 10^{34} \mathrm{erg} \mathrm{s}^{-1}$, non-dipole magnetic field components should be dominant at the emission region. For the $\gamma$-ray pulsars with $L_{\text {sd }} \lesssim 10^{35} \mathrm{erg} \mathrm{s}^{-1}$, observed $\gamma$-ray to X-ray and optical flux ratios are much higher than the flux ratio between curvature and the synchrotron radiations. We discuss some possibilities such as the coexistence of multiple accelerators in the magnetosphere as suggested from the recent numerical simulation results. The obtained maximum luminosity would be useful to select observational targets in X-ray and optical bands.
\end{abstract}

Subject headings: ——

\section{INTRODUCTION}

Rotation-powered pulsars are capable of producing pulsed emissions with wide energy bands. In some regions where the charge density is depleted from the Goldreich-Julian (GJ) charge density (Goldreich \& Julian 1969), particles are accelerated and emit high-energy photons. High-energy photons convert to electron and positron pairs which screen the accelerating electric field. Then, the particle acceleration and emission regions are spatially limited, which is of the pulsed emission (e.g., Sturrock 1971; Arons \& Scharlemann 1979; Cheng. Ho \& Ruderman 1986). During the processes, a part of the rotationenergy flux of a neutron star $L_{\text {sd }}$ converts to the particle kinetic energy flux. The primary particles which are significantly accelerated by the electric field emit the curvature radiation, and the created secondary (and higher generation) pairs with non-zero pitch angle emit synchrotron radiation. Thus, observations of pulsed emissions provide the location of particle acceleration and particle creation, and the efficiency of the energy conversion to the particle energy.

Pulsed emission in $\mathrm{GeV} \gamma$-ray band is an important tool for probing the particle acceleration in the magnetosphere. $\mathrm{GeV} \gamma$-ray emission is detected in young pulsars with characteristic age $\tau_{c} \lesssim 10^{6} \mathrm{yr}$, except for millisecond pulsars (Abdo et al. 2013). The emission

kisaka@phys.aoyama.ac.jp

sjtanaka@center.konan-u.ac.jp

${ }^{i}$ Department of Physics and Mathematics, Aoyama Gakuin

University, Sagamihara, Kanagawa, 252-5258, Japan

2 JSPS Research Fellow

3 Department of Physics, Konan University, Kobe, Hyogo, 658-8501, Japan mechanism is considered as the curvature radiation from the primary particles. Since the GeV spectrum observed by Fermi show that the cutoffs are more gradual than exponential (Abdo et al. 2009, 2013), the emission comes from the outer region of the magnetosphere such as the outer gap model (e.g., Cheng. Ho \& Ruderman 1986). The observed cutoff energy is distributed around $\sim \mathrm{GeV}$ (Abdo et al. 2013), which provides the energy of the accelerated particles. The observed $\gamma$-ray luminosity roughly seems to follow the trend $L_{\gamma} \propto L_{\mathrm{sd}}^{1 / 2}$ (Abdo et al. 2013). The trend is expected in models that the $\gamma$-ray luminosity is simply proportional to the GJ current (Harding 1981).

Although the $\gamma$-ray observations provide the constraints on the energy conversion efficiency and the location of the particle acceleration, the information could be inadequate to understand the particle acceleration for the whole population of rotation-powered pulsars. Since $\mathrm{GeV} \gamma$-ray emissions are detected from only young pulsars, the efficiency of energy conversion is less constrained for older, non- $\gamma$-ray pulsars. It is difficult to obtain the reliable value of the $\gamma$-ray luminosity of the $\gamma$-ray pulsars that are not detected in radio because of the uncertain distance from the observer. In addition, the energy conversion efficiency may be underestimated by only $\gamma$-ray observations because of the pair creation process. For example, the inner accelerator could also work to supply huge number of pairs required in the observations of pulsar wind nebulae (e.g., Tanaka \& Takahara 2010, 2011, 2013). The $\gamma$-ray photons from the inner region are significantly absorbed via magnetic $(\mathrm{B} \gamma)$ pair creation process, so that total energy flux radiated in $\gamma$-ray is larger than that estimated from the $\gamma$-ray observations.

The non-thermal pulsed emissions are also detected 
in lower energy bands such as X-ray and optical bands. The non-thermal X-ray luminosity seems to follow the relation $L_{\mathrm{X}} \propto L_{\mathrm{cd}}^{a}$ with $a \sim 0.9-1.5$ (e.g., Seward \& Wang 1988; Becker \& Trümper 1997; Possenti et al. 2002; Becker 2009), although scatter about the relation is significant (Kargaltsev et al. 2012; Shibata et al. 2016). Critical lines below which all the data locates on $L_{\mathrm{X}}$ versus $L_{\mathrm{sd}}$ plane are empirically suggested (Possenti et al. 2002; Kargaltsev et al. 2012), although the physical mechanism of the restriction of $\mathrm{X}$-ray luminosity is unknown. The non-thermal optical luminosity also seems to depend on the spin-down luminosity in a manner similar to the X-ray luminosity (e.g., Zharikov, Shibanov \& Komarova 2006). The emission mechanism for both X-ray and optical bands is widely believed as the synchrotron radiation from the secondary (and higher generation) particles (e.g., Romani 1996; Zhang \& Cheng 1997; Harding et al. 2008; Takata, Chang \& Shibata 2008).

The X-ray and optical emissions, and the combination with $\gamma$-ray observations provide valuable information about the particle acceleration. In contrast to $\mathrm{GeV} \gamma$-ray, the X-ray and optical emissions are detected even from old pulsars whose ages are up to $\sim 10^{8}$ yr (Posselt et al. 2012a). A photon whose energy is much less than a electron rest mass energy is not absorbed via the magnetic pair creation process. Then, we can detect the X-ray and optical emissions even if their emission regions reside at the inner region of the magnetosphere. These emissions provide the energy flux of secondary particles created in the magnetosphere for almost all pulsars. Since $\gamma$-ray, $\mathrm{X}$-ray and optical emitting particles are related through a pair cascade process, if the $\gamma$-ray emission is detected, their flux ratios which do not depend on the distance are useful to understand the pair cascade process in the magnetosphere.

One of the reasons for the investigation of the energy conversion for old pulsars and the comparison with that for young pulsars is that some of old pulsars have intensity and pulse profile modulations such as nulling and mode changing in radio observations (e.g., Backer 1970a, b). The fraction of these modulated pulsars significantly increases from their characteristic age $\tau_{\mathrm{c}} \gtrsim 10^{6} \mathrm{yr}$ (e.g., Wang. Manchester \& Johnston 2007), although it is unknown whether the characteristic age is a control parameter for the modulation phenomena or not. Recently, the correlation between the state changes of radio emission and the change of braking properties have been discovered (Kramer et al. 2006; Lyne et al. 2010). This correlation suggests that the modulation phenomena are linked and caused by the change of the magnetospheric state. Moreover, the synchronous radio and X-ray switching between two modes was also reported (Hermsen et al. 2013; Mereghetti et al. 2013, 2016). Therefore, the differences of the location of the X-ray emission region and the energy conversion efficiency between young and old pulsars give hints to understand such modulation phenomena.

Recently, enegy dissipation in the magnetosphere has been investigated by numerical simulations such as dissipative magnetohydrodynamic approaches (Li. Spitkovsky \& Tchekhovskov 2012; $\quad$ Kalapotharakos et al. 2012b;
Kalapotharakos, Harding \& Kazanas 2014) and global Particle-in-Cell simulations (Philippov \& Spitkovskv 2014; Chen \& Beloborodov 2014; Belvaev 2015; Philippov, Spitkovskv \& Cerutti 2015; Philippov et al. 2015; Cerutti et al. 2015; Cerutti, Philippov \& Spitkovsky 2016). Their results indicate that most particle acceleration takes place in the current sheet close behind the light cylinder (Cerutti \& Beloborodov 2016). The X-ray and optical synchrotron radiations from pairs in the current sheet have been discussed (e.g., Lyubarskii 1996). Hence, the X-ray and optical emissions would provide valuable constraints on the current sheet emitting scenario.

In this paper, using the analytical model, we calculate the luminosity of the synchrotron radiation as the emission mechanism of the non-thermal X-ray and optical bands. We parameterize the efficiency of energy conversion from the spin-down luminosity to the energy flux of primary particles. We are interested in the efficiency of the energy conversion and the emission region in whole population including $\gamma$-ray detected pulsars $\left(\tau_{\mathrm{c}} \lesssim 10^{6} \mathrm{yr}\right)$ and old pulsars $\left(\tau_{\mathrm{c}} \gtrsim 10^{6} \mathrm{yr}\right)$. In $\gamma$-ray pulsars, pair creation occurs at the outer magnetosphere via two-photon collision $(\gamma \gamma)$. As pulsar gets old, it is suggested that $\gamma \gamma$ pair creation becomes ineffective at the outer magnetosphere Wang \& Hirotani 2011; Kisaka \& Tanaka 2014). Thus, we consider two pair creation processes, $\mathrm{B} \gamma$ and $\gamma \gamma$ pair creations. In previous study (Kisaka \& Tanaka 2014), we assume that the energy of the primary particles equals to the maximum potential drop across the polar cap. Using this assumption, we avoid assuming the value of accelerating electric field which is highly uncertain. For young pulsars, the characteristic energy of the curvature radiation from such primary particles is much higher than the observed cutoff energy. Here, we use the typical value of the observed cutoff energy in $\mathrm{GeV} \gamma$-ray band to derive the energy of primary particles in $\gamma \gamma$ pair creation case. In $\mathrm{B} \gamma$ pair creation case, we use the maximum potential drop across the polar cap to derive the energy of primary particles. Note that in this case, the results do not depend on the energy of primary particles in our model. In section 2, we describe our model for the radiative transfer and the luminosity of the synchrotron radiation. In section 3. we provide the luminosity of the synchrotron radiation as a function of the efficiency of the energy conversion for each pair creation case. We also provide the allowed range of the emission region of the synchrotron radiation in X-ray and optical bands. Discussion is presented in section 4

\section{MODEL}

\subsection{Assumptions}

We consider synchrotron radiation emitted from created pairs via $\gamma \gamma$ or $\mathrm{B} \gamma$ pair creation process in the magnetosphere. In this paper, we use the term 'primary particles' as electrons or positrons that are accelerated in the magnetosphere and emit curvature photons whose energy is high enough to convert pairs. We also use 'secondary particles' as electrons and positrons created outside of the acceleration region and emit synchrotron radiation. Since we are interested in particles which emit the synchrotron radiation, 'secondary particles' include second 


\section{YY scenario}

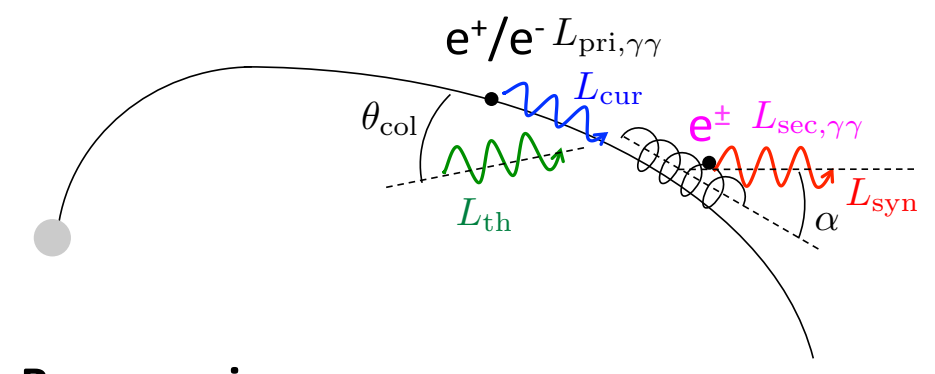

\section{By scenario}

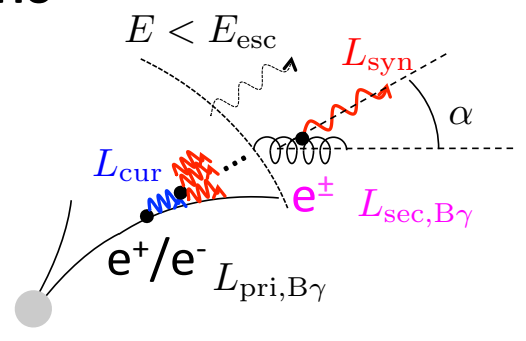

FIG. 1.- Schematic pictures of our model for $\gamma \gamma$ (upper panel) and B $\gamma$ scenarios (lower panel). Grey circles are neutron stars, and thin solid lines denote the magnetic field lines. Blue, green, and red arrows denote the photons of curvature radiation from primary particle, thermal radiation from heated polar cap, and synchrotron radiation from secondary (and higher generation) pairs. The definitions of the energy fluxes for the primary and secondary particles in $\gamma \gamma$ scenario are $L_{\mathrm{pri}, \gamma \gamma} \equiv \min \left\{\gamma_{\mathrm{p}, \mathrm{E}}, \gamma_{\mathrm{p}, \max }\right\} m_{\mathrm{e}} c^{2} \dot{N}_{\mathrm{p}}$ and $L_{\mathrm{sec}, \gamma \gamma} \equiv 2 \gamma_{\mathrm{s}, \mathrm{syn}} m_{\mathrm{e}} c^{2} \dot{N}_{\mathrm{p}} N_{\gamma} \tau_{\gamma \gamma}$, respectively. On the other hand, in B $\gamma$ scenario, the energy fluxes of the primary and secondary particles are $L_{\mathrm{pri}, \mathrm{B} \gamma} \equiv \gamma_{\mathrm{p}, \mathrm{max}} m_{\mathrm{e}} c^{2} \dot{N}_{\mathrm{p}}$, and $L_{\mathrm{sec}, \mathrm{B} \gamma} \equiv 2 \gamma_{\mathrm{s}, \mathrm{syn}} m_{\mathrm{e}} c^{2} \dot{N}_{\mathrm{p}} N_{\gamma} E_{\mathrm{cur}} / E_{\mathrm{esc}}$, respectively. See text details.

and higher generation particles which could emit synchrotron radiation.

In our model, we make three main assumptions that (a) the physical quantities at the regions of the particle acceleration, creation and emission are described as a function of the radius $r$ from the centre of the neutron star to the radius of the light cylinder $R_{\mathrm{lc}}$, (b) the energy source is only the rotational energy of a neutron star and other sources such as the magnetic field dissipation do not contribute to the particle energy flux, and (c) the dipole component of the magnetic field dominates near the light cylinder, where the radius of the light cylinder is

$$
R_{\mathrm{lc}}=\frac{P c}{2 \pi},
$$

where $c$ is the speed of light. Note that although we only consider the region inside the light cylinder as in assumption (a), the model with $r \sim R_{\mathrm{lc}}$ is applicable to the the case where significant dissipation of Poynting flux takes place close behind the light cylinder $\left(\sim 1-2 R_{\mathrm{lc}}\right)$ as indicated by recent global models (Belvaev 2015; Cerutti et al. 2015). Moreover, the synchrotron luminosity becomes maximum at $R=R_{\mathrm{lc}}$ if our model extends outside the light cylinder as discussed in section 4 . From assumption (b), the total energy flux of primary particles is limited by the spin-down luminosity. We introduce the dimensionless conversion efficiency $\eta(\leq 1)$ from the spin-down luminosity $L_{\mathrm{sd}}$ to the energy flux of the primary particles as a model parameter (see also equation 16).

The radius, mass and dipole magnetic moment of a neutron star are $R_{\mathrm{ns}}=10^{6} \mathrm{~cm}, M_{\mathrm{ns}}=1.4 M_{\odot}$, and $\mu_{\text {mag }}^{2} \equiv\left(B_{\mathrm{s}} R_{\mathrm{ns}}^{3} / 2\right)^{2}=3 I c^{3} P \dot{P} / 8 \pi^{2}$, respectively, where $I=(2 / 5) M_{\mathrm{ns}} R_{\mathrm{ns}}^{2}$ is moment of inertia. For the $\gamma \gamma$ pair creation process, we consider the thermal X-ray photons from the heated polar cap as the seed photons to create the secondary pairs, while we neglect the thermal photons from the entire surface and the synchrotron photons. This is reasonable approximation except for very young pulsar such as the Crab pulsar (e.g., Yakovlev \& Pethick 2004). The synchrotron luminosity in ingoing case, where synchrotron emitting pairs are created by the curvature photons emitted by the ingoing primary particles, is lower than the luminosity in outgoing case as shown in previous studies (Kisaka \& Tanaka 2014, 2015). We only consider the synchrotron radiation from pairs created by the curvature photons emitted by the outgoing primary particles.

We consider two pair creation processes, $\gamma \gamma$ and $\mathrm{B} \gamma$ pair creations, separately. The schematic pictures of two scenarios are shown in figure 1. In $\gamma \gamma$ pair creation scenario (upper panel), the curvature photons (blue arrow) collide with the thermal photons (green arrow) from the heated polar cap. Then, the secondary electrons and positrons are created and emit the synchrotron radiation (red arrow). The particle acceleration and $\gamma \gamma$ pair creation regions reside near the light cylinder as is considered in the outer gap model (e.g., Cheng. Ho \& Ruderman 1986). The thermal emission from the heated polar cap are detected in soft X-ray observations (e.g., Halpern \& Ruderman 1993; Zavlin \& Pavlov 2004), so that we can easily estimate the optical depth for $\gamma \gamma$ pair creation. Since the optical 
depth for the propagation of curvature photons is typically much lower than unity (see section 2.2) at the outer magnetosphere, most curvature photons escape from the magnetosphere. These pulsars are detected as $\gamma$-ray pulsars (e.g., Abdo et al. 2013). In $\gamma \gamma$ scenario, we use the typical observed energy of $\gamma$-ray pulsars $E_{\text {cur }}$ as a parameter, to obtain the Lorentz factor of the primary particles as,

$$
\gamma_{\mathrm{p}, \mathrm{E}} \equiv \gamma_{\mathrm{p}}\left(E_{\mathrm{cur}}\right)=\left(\frac{4 \pi}{0.87} \frac{E_{\mathrm{cur}}}{h} \frac{R_{\mathrm{cur}}}{c}\right)^{1 / 3},
$$

where $R_{\text {cur }}$ is the curvature radius, and $h$ is the Planck constant. The Lorentz factor $\gamma_{\mathrm{p}, \mathrm{E}}$ should be lower than the maximum Lorentz factor determined by the full potential drop across the polar cap surface,

$$
\gamma_{\mathrm{p}, \max }=\frac{2 \pi^{2} e B_{\mathrm{s}} R_{\mathrm{ns}}^{3}}{m_{\mathrm{e}} c^{4} P^{2}},
$$

where $m_{\mathrm{e}}$ and $e$ is the mass and the charge of an electron (Goldreich \& Julian 1969). Thus, we use $\gamma_{p}=\min \left\{\gamma_{p, E}, \gamma_{p, \max }\right\}$ as the Lorentz factor of the primary particles. Note that even if we consider the non-dipole components such as toroidal and higher order poloidal components near the surface as considered in some authors (e.g., Ruderman \& Sutherland 1975; Geppert \& Viganò 2014; Szary, Melikidze \& Gil 2015), the potential drop across the polar cap is determined by the dipole magnetic field as long as assumption (c) is satisfied. Only low- $L_{\text {sd }}$ pulsars $\left(L_{\text {sd }} \lesssim 10^{34}\right.$ erg s $\left.^{-1}\right)$ satisfy the condition $\gamma_{\mathrm{p}, \max }<\gamma_{\mathrm{p} \text {, E }}$ with $E_{\text {cur }} \sim \mathrm{GeV}$. The energy of primary particles could reach almost whole potential drop for such low- $L_{\text {sd }}$ pulsars (Takata \& Chang 2009).

In $\mathrm{B} \gamma$ pair creation scenario (lower panel in figure 1), the pair creation occurs near the stellar surface via the interaction between the photons and the strong magnetic field. The curvature (blue arrow) and high-energy synchrotron photons (red arrows) convert to pairs in $\mathrm{B} \gamma$ process. Since most of curvature photons emitted from the primary particles are efficiently absorbed via $\mathrm{B} \gamma$ pair creation process (e.g., Daugherty \& Harding 1982), equation (2) is not available to estimate the Lorentz factor of the primary particles $\gamma_{\mathrm{p}}$. We use the maximum Lorentz factor $\gamma_{\mathrm{p} \text {,max }}$ in equation (3) as the Lorentz factor of the primary particles, although the synchrotron luminosity does not depend on the Lorentz factor of primary particles for most pulsars in our model as discussed in section 3. For the cascade at the inner region, we assume that all photons with energy higher than the energy $E_{\text {esc }}$ converts to pairs, where $E_{\text {esc }}$ is the maximum escapable photon energy and is described as a function of $r$ (see equation 15). The maximum number of photons which convert to pairs is about $E_{\text {cur }} / E_{\text {esc }}$ times larger than the number of curvature photons with energy $E_{\text {cur }}$ from the primary particles. This is an ideal cascade case considered in Kisaka \& Tanaka (2014); Timokhin \& Harding (2015). We use this number of photons to estimate the number of the synchrotron emitting particles (see equation [12).

In $\mathrm{B} \gamma$ scenario, we consider two cases for the magnetic field near the stellar surface. First, only the dipole component dominates at the entire magnetosphere. Second, the non-dipole components significantly contribute to the magnetic field at the emission region. In the latter case, we use the simple description to the magnetic field at the emission region as,

$$
B \equiv \zeta_{\mathrm{B}} B_{\mathrm{s}}\left(r / R_{\mathrm{ns}}\right)^{-3},
$$

where $\zeta_{\mathrm{B}}(\geq 1)$ is a model parameter. Equation (4) reduces to dipole field in the limit $\zeta_{\mathrm{B}}=1$. The configuration of the magnetic field has also effects on the curvature radius of the field line $R_{\text {cur }}$ and the pitch angle of secondary particles $\alpha$ in our model. We use the following approximated formula (e.g., Tang et al. 2008; Kisaka \& Tanaka 2014),

$$
R_{\text {cur }} \sim \begin{cases}\sqrt{r R_{\mathrm{lc}}} & (\text { dipole }) \\ r & (\text { non }- \text { dipole })\end{cases}
$$

and

$$
\alpha \sim \begin{cases}\sqrt{r / R_{\mathrm{lc}}} & (\text { dipole }) \\ \alpha_{0} & (\text { non }- \text { dipole })\end{cases}
$$

where the pitch angle $\alpha_{0}(\leq 1)$ is a model parameter.

\subsection{Analytical Description of Synchrotron Luminosity}

In our model, the newly created secondary particles in the magnetosphere have non-zero pitch angle $\alpha$ (equation 6). The secondary particles emit synchrotron radiation. Taking into account the radiation cooling effect, the effective number of the synchrotron emitting particles at the emission region $r$ is $\sim \dot{N}_{\mathrm{s}} \times \min \left\{t_{\mathrm{ad}}, t_{\text {cool,syn }}\right\}$, where $\dot{N}_{\mathrm{s}}$ is the total number flux of the secondary particles, and two timescales $t_{\text {ad }}$ and $t_{\text {cool,syn }}$ are the advection and the synchrotron cooling timescales, respectively. The advection timescale at the emission region $r$ is given by

$$
t_{\mathrm{ad}} \sim \frac{r}{c}
$$

and the cooling timescale of synchrotron radiation with the characteristic energy $h \nu_{\mathrm{obs}}$ is given by

$$
t_{\mathrm{cool}, \mathrm{syn}} \sim \frac{\gamma_{\mathrm{s}, \mathrm{syn}} \alpha m_{\mathrm{e}} c^{2}}{P_{\mathrm{syn}}},
$$

where $P_{\text {syn }}$ is the power of the synchrotron radiation for each particle 4 ,

$$
P_{\text {syn }}=\frac{2 e^{4} B^{2} \alpha^{2}}{3 c^{3} m_{\mathrm{e}}^{2}} \gamma_{\mathrm{s}, \mathrm{syn}}^{2},
$$

and $\gamma_{\mathrm{s} \text { syn }}$ is the Lorentz factor of the secondary particles which emit the synchrotron radiation with the characteristic energy $h \nu_{\mathrm{obs}}$,

$$
\gamma_{\mathrm{s}, \mathrm{syn}}=\sqrt{\frac{4 \pi}{0.87} \nu_{\mathrm{obs}} \frac{m_{\mathrm{e}} c}{e B \alpha}} .
$$

Using the effective number of secondary particles and the synchrotron power for each particle, the luminosity

4 This classical formula for synchrotron power is not valid for $\gamma \alpha B / B_{\mathrm{q}}>0.1$ (e.g., Harding \& Lai 2006). However, we consider the characteristic energy of synchrotron radiation, which is much lower than the electron rest mass energy so that condition $\gamma \alpha B / B_{\mathrm{q}} \ll 0.1$ is satisfied. 
of the synchrotron radiation from the secondary pairs is described as,

$$
L_{\text {syn }} \sim P_{\text {syn }} \dot{N}_{\mathrm{s}} \min \left\{t_{\mathrm{ad}}, t_{\mathrm{cool}, \mathrm{syn}}\right\} .
$$

We model the radiative transfer for the curvature photons analytically to calculate the number flux of the secondary pairs, $\dot{N}_{\mathrm{s}}$. The number flux of the secondary pairs in $\gamma \gamma$ and $\mathrm{B} \gamma$ scenarios is described by

$$
\dot{N}_{\mathrm{s}} \sim 2 \dot{N}_{\mathrm{p}} N_{\gamma} \times \begin{cases}\min \left\{\tau_{\gamma \gamma}, 1\right\} & (\gamma \gamma), \\ \frac{E_{\mathrm{cur}}}{E_{\mathrm{esc}}} & (\mathrm{B} \gamma),\end{cases}
$$

where $\dot{N}_{\mathrm{p}}$ is the total number fux of the primary particles, $N_{\gamma}$ is the number of curvature photons emitted from one primary particle, and $\tau_{\gamma \gamma}$ is the optical depth for $\gamma \gamma$ pair creation. The factor 2 on the right-hand side of equation (12) accounts for electron and positron. The optical depth for the curvature photons in $\gamma \gamma$ pair creation process is given by

$$
\tau_{\gamma \gamma} \sim \frac{L_{\mathrm{pc}}}{4 \pi r^{2} c E_{\mathrm{pc}}} \sigma_{\gamma \gamma}\left(1-\cos \theta_{\mathrm{col}}\right) r,
$$

where $L_{\mathrm{pc}}$ is the luminosity of the thermal emission from the heated polar cap, $E_{\mathrm{pc}}=2.82 k T_{\mathrm{pc}}$ and $T_{\mathrm{pc}}$ are the energy and temperature of the thermal photons, $k$ is Boltzmann constant, $\sigma_{\gamma \gamma} \sim 0.2 \sigma_{\mathrm{T}}$ and $\sigma_{\mathrm{T}}$ are the cross sections for the $\gamma \gamma$ pair creation and the Thomson scattering, and $\theta_{\mathrm{col}}$ is the collision angle of the curvature and thermal photons, which approximately described by

$$
1-\cos \theta_{\text {col }} \sim \frac{1}{2}\left(\frac{r}{R_{\text {cur }}}\right)^{2} \sim \frac{1}{2}\left(\frac{r}{R_{\mathrm{lc}}}\right) .
$$

The soft X-ray observations suggest that the thermal luminosity from the heated polar cap $L_{\mathrm{pc}}$ is approximately proportional to the spin-down luminosity, $L_{\mathrm{pc}} \sim$ $10^{-3} L_{\text {sd }}$ (Becker \& Trümper 1997; Becker 2009). We use the normalized luminosity $\epsilon_{\mathrm{pc}} \equiv L_{\mathrm{pc}} / L_{\mathrm{sd}}$ instead of $L_{\mathrm{pc}}$. For typical $\gamma$-ray pulsars, the optical depth is $\tau_{\gamma \gamma} \sim 2.7 \times 10^{-4}\left(\epsilon_{\mathrm{pc}} / 10^{-3}\right)\left(T_{\mathrm{pc}} / 10^{6} \mathrm{~K}\right)^{-1}(P / 0.1 \mathrm{~s})^{-1}$, much smaller than unity. Only a part of the curvature photons converts to the pairs. On the other hand, in $\mathrm{B} \gamma$ pair creation, because of the high efficiency of the pair conversion, we assume that all photons with energy higher than $E_{\text {esc }}$ convert to pairs in the electromagnetic cascade (Kisaka \& Tanaka 2014; Timokhin \& Harding 2015). The maximum energy of the escapable photon is described by (e.g., Erber 1966)

$$
E_{\mathrm{esc}}=2 m_{\mathrm{e}} c^{2} \chi_{\min } \frac{B_{\mathrm{q}}}{B_{\perp}},
$$

where the magnetic fields $B_{\mathrm{q}}=m_{\mathrm{e}}^{2} c^{3} / e \hbar \sim 4.4 \times 10^{13} \mathrm{G}$ and $B_{\perp}=B \sin \theta_{\mathrm{B} \gamma}$, and we take the critical value as $\chi_{\min }=1 / 15$ following Ruderman \& Sutherland (1975) 5. The angle $\theta_{\mathrm{B} \gamma}$, which is the angle between the direction of the propagation for a curvature photon and

5 Equation (15) is not valid for $B>0.1 B_{\mathrm{q}}$ (e.g., Daugherty \& Harding 1983). However, from inequality (23) discussed in section 2.3 the magnetic field is limited $B \lesssim 0.1 \alpha B_{\mathrm{q}}$ at the emission region as long as we consider the energy range $h \nu_{\text {obs }} \lesssim 50 \mathrm{keV}$. the magnetic field, is comparable to the pitch angle of secondary pairs (equation 6). Then, we use the approximation $\theta_{\mathrm{B} \gamma} \sim \alpha$.

We model the primary particles that emit the curvature radiation to estimate the number flux $\dot{N}_{\mathrm{p}}$ and the number of curvature photons $N_{\gamma}$. For the total number flux of the primary particles $\dot{N}_{\mathrm{p}}$ with the Lorentz factor $\gamma_{\mathrm{p}}$, we introduce a parameter $\eta$ which corresponds to the conversion efficiency from the spin-down luminosity $L_{\mathrm{sd}}$ as

$$
\dot{N}_{\mathrm{p}}=\frac{\eta L_{\mathrm{sd}}}{\gamma_{\mathrm{p}} m_{\mathrm{e}} c^{2}} .
$$

From assumption (b) in section 2.1, the efficiency must be smaller than unity, $\eta \leq 1$. In order to estimate the number of curvature photons for a particle $N_{\gamma}$, we consider two timescales, the advection $t_{\text {ad }}$ (equation 7) and the curvature cooling timescales $t_{\text {cool,cur. The curvature }}$ cooling timescale is given by

$$
t_{\text {cool,cur }} \sim \frac{\gamma_{\mathrm{p}} m_{\mathrm{e}} c^{2}}{P_{\text {cur }}},
$$

where $P_{\text {cur }}$ is the power of curvature radiation from a particle,

$$
P_{\text {cur }}=\frac{2 e^{2} c}{3 R_{\text {cur }}^{2}} \gamma_{\mathrm{p}}^{4}
$$

The number of the curvature photons for a particle $N_{\gamma}$ is estimated by

$$
N_{\gamma} \sim \frac{P_{\text {cur }}}{E_{\text {cur }}} \min \left\{t_{\text {cool,cur }}, t_{\text {ad }}\right\} .
$$

Substituting equations (9), (10), (12 - 19) into equation (11), we obtain the luminosity of the synchrotron radiation as a function of $r$. For the derived values, model parameters is only $\eta$ in $\gamma \gamma$ and $\mathrm{B} \gamma$ scenario in the dipole dominant case. If we take into account the effects of the non-dipole field for the strength of the local magnetic field (equation 4), the pitch angle (equation 6), and the curvature radius (equation 51) in $\mathrm{B} \gamma$ scenario, the derived synchrotron luminosity also depends on the additional model parameters, $\alpha_{0}$ and $\zeta_{\mathrm{B}}$. The values of $\nu_{\mathrm{obs}}, E_{\mathrm{cur}}, \epsilon_{\mathrm{pc}}, T_{\mathrm{pc}}, B_{\mathrm{s}}$ and $L_{\mathrm{sd}}$ are taken from the observations.

\subsection{Constraints on Emission Region}

There are some constraints on the emission region $r$ in our synchrotron radiation model. We assume that the emission region resides in the magnetosphere,

$$
R_{\mathrm{ns}}<r<R_{\mathrm{lc}} .
$$

In addition, we consider following four conditions in order to constrain the emission region $r$.

First condition is that the Lorentz factor of the created secondary particles from the curvature photons $\gamma_{\mathrm{s} \text {,pair }}$ has to be larger than the Lorentz factor of particles $\gamma_{\mathrm{s}, \mathrm{syn}}$ which emit the synchrotron radiation with the characteristic energy $h \nu_{\mathrm{obs}}$,

$$
\gamma_{\mathrm{s}, \text { pair }}>\gamma_{\mathrm{s}, \mathrm{syn}}
$$


where $\gamma_{\text {s,pair }}$ is the Lorentz factor of the secondary pairs from a photon energy $E_{\text {cur }}$,

$$
\gamma_{\mathrm{s}, \text { pair }}=\frac{E_{\mathrm{cur}}}{2 m_{\mathrm{e}} c^{2}} \text {. }
$$

Since the Lorentz factor for a given frequency $\nu_{\mathrm{obs}}$ is $\gamma_{\mathrm{s}, \mathrm{syn}} \propto r^{3 / 2} \alpha^{-1 / 2}$ (equation [10), energy condition (21) gives the upper limit on the emission region, $r \leq r_{\gamma s y n}$.

Second condition is the validity of the synchrotron approximation, $\gamma_{\mathrm{s}, \mathrm{syn}} \alpha>1$. The condition indicates that the observed frequency $\nu_{\text {obs }}$ should be higher than the low-energy turnover frequency for the synchrotron radiation (O’Dell \& Sartori 1970; Rudak \& Dyks 1999),

$$
\nu_{\mathrm{obs}}>\frac{e B}{2 \pi m_{\mathrm{e}} c \alpha} \text {. }
$$

Condition (23) gives the lower limit on the emission region, $r \geq r_{\mathrm{ct}}$.

Third and fourth conditions come from the pair creation thresholds. The $\gamma \gamma$ pair creation threshold is described by

$$
\left(1-\cos \theta_{\mathrm{col}}\right) E_{\mathrm{pc}} E_{\mathrm{cur}}>2\left(m_{\mathrm{e}} c^{2}\right)^{2} .
$$

Under the dipole magnetic field configuration, the collision angle $\theta_{\text {col }}$ becomes large toward the large distance from the neutron star (equation 14). Then, condition (24) gives the lower limit, $r \geq r_{\gamma \gamma}$. The $\mathrm{B} \gamma$ pair creation threshold is described by (Erber 1966)

$$
\frac{E_{\text {cur }}}{2 m_{\mathrm{e}} c^{2}} \frac{B_{\perp}}{B_{\mathrm{q}}}>\chi_{\mathrm{min}} .
$$

Since the occurrence of the $\mathrm{B} \gamma$ pair production requires the strong magnetic field, condition (25) gives upper limit $r \leq r_{\mathrm{B} \gamma}$.

From conditions (20,24), the range of the emission region in $\gamma \gamma$ pair creation scenario is

$$
\max \left\{R_{\mathrm{ns}}, r_{\mathrm{ct}}, r_{\gamma \gamma}\right\}<r<\min \left\{R_{\mathrm{lc}}, r_{\gamma \mathrm{syn}}\right\} .
$$

Within typical parameter ranges, the relation $\max \left\{R_{\mathrm{ns}}, r_{\mathrm{ct}}, r_{\gamma \gamma}\right\}=r_{\gamma \gamma}$ would be satisfied. Then, the allowed range of the emission region is $r_{\gamma \gamma}<r<\min \left\{R_{\mathrm{lc}}, r_{\gamma \mathrm{syn}}\right\}$ for most pulsars.

In $\mathrm{B} \gamma$ pair creation scenario, the range of the emission region is

$$
\max \left\{R_{\mathrm{ns}}, r_{\mathrm{ct}}\right\}<r<\min \left\{R_{\mathrm{lc}}, r_{\gamma \mathrm{syn}}, r_{\mathrm{B} \gamma}\right\}
$$

from conditions (20,23) and (25). Within the typical ranges of the parameters, the relations $\max \left\{R_{\mathrm{ns}}, r_{\mathrm{ct}}\right\}=$ $r_{\mathrm{ct}}, r_{\gamma \mathrm{syn}}>R_{\mathrm{lc}}$, and $r_{\gamma \mathrm{syn}}>r_{\mathrm{B} \gamma}$ would be satisfied. Then, the range of the emission region is $r_{\text {ct }}<r<$ $\min \left\{R_{\mathrm{lc}}, r_{\mathrm{B} \gamma}\right\}$.

\section{RESULTS}

In this section, we calculate the luminosity of the synchrotron radiation based on the model described in previous section. In section 3.1, we derive the efficiency of the synchrotron radiation relative to the spin-down luminosity as a function of $r$ from equation (11). In section 3.2 we provide the allowed range of the emission region $r$ and the death lines for each scenario from the constraints described in section 2.3. Using the efficiency of the synchrotron radiation as a function of $r$ and the constraints on $r$, we provide the maximum luminosity in the allowed emission region and its dependence on the spin-down luminosity in section 3.3 . Comparing with the observed non-thermal luminosity, we provide the required range of the efficiency parameter $\eta$. In section 3.4 we derive the ratio of the curvature to the synchrotron luminosities, which do not depend on the distance from the observer and the model parameter $\eta$. Then, we compare with the flux ratios of observed $\gamma$-ray to X-ray and optical. Hereafter, we use $Q_{x} \equiv Q / 10^{x}$ in cgs units, $E_{\text {cur }, \mathrm{GeV}} \equiv E_{\text {cur }} / 1$ $\mathrm{GeV}$, and $h \nu_{\mathrm{keV}} \equiv h \nu / 1 \mathrm{keV}$.

\subsection{Synchrotron Efficiency}

Before the detailed calculations, we see the main control parameters of the efficiency of the synchrotron radiation $\epsilon_{\mathrm{syn}} \equiv L_{\mathrm{syn}} / L_{\mathrm{sd}}$ in the conditions where the radiative cooling timescales are smaller than the advection timescale in equations (12) and (19) $\left(t_{\text {cool,syn }}<t_{\mathrm{ad}}\right.$ and $\left.t_{\text {cool,cur }}<t_{\text {ad }}\right)$.

In $\gamma \gamma$ pair creation scenario, the efficiency $\epsilon_{\mathrm{syn}}$ is derived from equation (11) as,

$$
\epsilon_{\mathrm{syn}} \sim \eta \tau_{\gamma \gamma} \frac{\gamma_{\mathrm{s}, \mathrm{syn}} \alpha}{\gamma_{\mathrm{s}, \mathrm{pair}}}
$$

Near the light cylinder, $r \sim R_{\mathrm{lc}}$, the pitch angle $\alpha$ becomes order unity (equation 6). For the typical $\gamma$-ray pulsars, the optical depth is $\tau_{\gamma \gamma} \sim 10^{-4}-10^{-2}$ (equation 13) and the energy ratio $\gamma_{\mathrm{s}, \mathrm{syn}} / \gamma_{\mathrm{s}, \mathrm{pair}} \sim(0.1-1) \nu_{\mathrm{obs}, \mathrm{keV}}^{1 / 2}$ (equations 10 and 22). Then, the efficiency of the synchrotron radiation is roughly $\epsilon_{\mathrm{syn}} \sim 10^{-5}-10^{-2} \eta \nu_{\mathrm{obs}, \mathrm{keV}}^{1 / 2}$ which is roughly consistent with observed non-thermal X-ray efficiency (e.g., Kargaltsev et al. 2012) if the conversion efficiency is an order of unity, $\eta \sim 1$.

In $\mathrm{B} \gamma$ pair creation scenario, the efficiency is

$$
\epsilon_{\mathrm{syn}} \sim \eta \frac{\gamma_{\mathrm{s}, \mathrm{syn}} \alpha m_{\mathrm{e}} c^{2}}{E_{\mathrm{esc}}}
$$

This efficiency does not depend on the Lorentz factor of primary particles $\gamma_{p}$, unless $\gamma_{p}$ is too low to create the pairs which emit synchrotron radiation. Near the stellar surface $r \sim R_{\mathrm{ns}}$, since the energy ratio is $\gamma_{\mathrm{s}, \mathrm{syn}} m_{\mathrm{e}} c^{2} / E_{\mathrm{esc}} \sim 10^{-2} \alpha^{1 / 2} \zeta_{\mathrm{B}}^{1 / 2} \nu_{\mathrm{obs}, \mathrm{keV}}^{1 / 2}$, the efficiency is $\epsilon_{\text {syn }} \sim 10^{-2} \eta \alpha^{3 / 2} \zeta_{\mathrm{B}}^{1 / 2} \nu_{\mathrm{obs}, \mathrm{keV}}^{1 / 2}$. If we consider the high conversion efficiency $\eta \sim 1$ and the non-dipole magnetic field with the pitch angle $\alpha \sim 1$, the synchrotron efficiency could be $\epsilon_{\mathrm{syn}} \sim 10^{-2} \zeta_{\mathrm{B}}^{1 / 2} \nu_{\mathrm{obs}, \mathrm{keV}}^{1 / 2}$.

The conditions, $t_{\text {cool,syn }}<t_{\text {ad }}$ and $t_{\text {cool,cur }}<t_{\text {ad }}$, are not always satisfied. Then, the synchrotron efficiency becomes reduced. In what follows, we calculate the efficiency of the synchrotron radiation comparing timescales $t_{\mathrm{ad}}, t_{\mathrm{cool}, \mathrm{syn}}$, and $t_{\mathrm{cool}, \mathrm{cur}}$. First, we compare the synchrotron cooling and advection timescales, $t_{\text {cool,syn }}$ and $t_{\text {ad }}$, to calculate the synchrotron luminosity $L_{\text {syn }}$ from equation (11). The normalized radius $r_{\text {eq,syn, } 6} \equiv r_{\text {eq,syn }} / 10^{6} \mathrm{~cm}$ where the synchrotron cooling timescale equals to the advection timescale, $t_{\text {cool,syn }}=$ 
$t_{\mathrm{ad}}$, is

$$
r_{\mathrm{eq}, \mathrm{syn}, 6} \sim\left\{\begin{array}{c}
8.6 \times 10^{2} \nu_{\mathrm{obs}, \mathrm{keV}}^{2 / 13} B_{\mathrm{s}, 12}^{11 / 26} L_{\mathrm{sd}, 31}^{1 / 52} \\
(\mathrm{dipole}), \\
9.7 \times 10^{2} \alpha_{0}^{1 / 7} \zeta_{\mathrm{B}}^{3 / 7} \nu_{\mathrm{obs}, \mathrm{keV}}^{1 / 7} B_{\mathrm{s}, 12}^{3 / 7} \\
(\text { non }- \text { dipole) } .
\end{array}\right.
$$

At the inner region $r<r_{\text {eq,syn }}$, we should consider the synchrotron cooling timescale $\left(t_{\text {cool,syn }}<t_{\text {ad }}\right)$ to calculate the luminosity $L_{\mathrm{syn}}$ from equation (11). In $\mathrm{B} \gamma$ scenario, the emission region resides near the stellar surface (section 2.1, figure 11), so that condition $r<r_{\text {eq,syn }}\left(t_{\text {cool,syn }}<t_{\text {ad }}\right)$ is satisfied. We only consider $\min \left\{t_{\mathrm{cool}, \mathrm{syn}}, t_{\mathrm{ad}}\right\}=t_{\mathrm{cool}, \mathrm{syn}}$ in $\mathrm{B} \gamma$ scenario. On the other hand, in $\gamma \gamma$ scenario, the emission region could reside at the outer region of the magnetosphere (section [2.1 figure (1), so that the emission region could reside $r>r_{\text {eq,syn }}\left(t_{\text {cool,syn }}>t_{\text {ad }}\right)$ for some pulsars with the low spin-down luminosity. Note that the radius $r_{\text {eq,syn }}$ in equation (30) does not depend on whether equation (2) or (3) is used as the Lorentz factor of the primary particles.

Next, we compare the curvature cooling timescale $t_{\text {cool,cur }}$ and advection timescale $t_{\mathrm{ad}}$ to calculate the number of $\gamma$-ray photons $N_{\gamma}$ from equation (19). Using the dipole magnetic field and the Lorentz factor of the primary particles $\gamma_{\mathrm{p}, \mathrm{E}}$ (equation 22), the condition $t_{\text {cool,cur }}<t_{\text {ad }}$ gives the lower limit $r>r_{\text {eq,cur }}$ on the radius,

$$
r_{\text {eq, cur }, 6} \sim 9.9 E_{\text {cur, GeV }}^{-2} B_{\mathrm{s}, 12}^{1 / 2} L_{\mathrm{sd}, 31}^{-1 / 4}
$$

$$
\left(\gamma_{\mathrm{p}, \mathrm{E}}, \text { dipole }\right) \text {. }
$$

In $\gamma \gamma$ scenario, the emission region resides near the light cylinder, $r \sim R_{\mathrm{lc}}$, so that $r>r_{\text {eq,cur }}\left(t_{\text {cool,cur }}<t_{\mathrm{ad}}\right)$ is satisfied for most pulsars. If we use the Lorentz factor $\gamma_{\mathrm{p}, \max }$ (equation 3), condition $t_{\mathrm{cool}, \mathrm{cur}}<t_{\mathrm{ad}}$ does not depend on the radius $r$. Condition $t_{\mathrm{cool}, \mathrm{cur}}<t_{\mathrm{ad}}$ constrains on the spin-down luminosity as,

$$
L_{\mathrm{sd}, 31} \gtrsim 4.0 B_{\mathrm{s}, 12}^{2 / 7} \quad\left(\gamma_{\mathrm{p}, \max }, \text { dipole }\right),
$$

which is satisfied for most pulsars. This condition is applicable to both $\gamma \gamma$ and $\mathrm{B} \gamma$ scenarios. On the other hand, if we consider the non-dipole components of the magnetic field at the emission region and use the Lorentz factor of the primary particles $\gamma_{\mathrm{p} \text {,max }}$ as considered in $\mathrm{B} \gamma$ scenario, condition $t_{\text {cool,cur }}<t_{\text {ad }}$ gives the upper limit $\left(r<r_{\text {eq,cur }}\right)$ on the radius,

$$
\begin{array}{r}
r_{\text {eq, cur }, 6} \sim 4.3 \times 10^{2} L_{\mathrm{sd}, 31}^{3 / 2} \\
\quad\left(\gamma_{\mathrm{p}, \max }, \text { non }- \text { dipole }\right) .
\end{array}
$$

Radius $r_{\text {eq,cur }}$ is much larger than the stellar radius $R_{\mathrm{ns}}$. In $\mathrm{B} \gamma$ scenario, the emission region resides near the stellar surface, $r \sim R_{\mathrm{ns}}$. Then, condition $r<r_{\text {eq,cur }}$ $\left(t_{\text {cool,cur }}<t_{\text {ad }}\right)$ at the emission region should be satisfied. From conditions (31) 33), we use $\min \left\{t_{\mathrm{cool}, \mathrm{cur}}, t_{\mathrm{ad}}\right\}=$ $t_{\text {cool,cur for most pulsars. }}$

Substituting equations (6- 10) and (12-19) into equation (11), we obtain the efficiencies of synchrotron radiation $\epsilon_{\mathrm{syn}}$ as a function of the radius $r$. In $\gamma \gamma$ pair creation scenario, the efficiencies are given by

$$
\epsilon_{\mathrm{syn}} \sim\left\{\begin{array}{c}
1.7 \times 10^{-14} \eta \nu_{\mathrm{obs}, \mathrm{keV}}^{1 / 2} E_{\mathrm{cur}, \mathrm{GeV}}^{-1} \\
\times \epsilon_{\mathrm{pc},-3} T_{\mathrm{pc}, 6.5}^{-1} B_{\mathrm{s}, 12}^{-9 / 8} L_{\mathrm{sd}, 31}^{21 / 16} r_{6}^{7 / 4} \\
\left(t_{\mathrm{cool}, \mathrm{syn}}<t_{\mathrm{ad}}, \gamma_{\mathrm{p}, \mathrm{E}}\right), \\
5.9 \times 10^{-14} \eta \nu_{\mathrm{obs}, \mathrm{keV}}^{1 / 2} \\
\times \epsilon_{\mathrm{pc},-3} T_{\mathrm{pc}, 6.5}^{-1} B_{\mathrm{s}, 12}^{-7 / 8} L_{\mathrm{sd}, 31}^{-5 / 16} r_{6}^{9 / 4} \\
\left(t_{\mathrm{cool}, \mathrm{syn}}<t_{\mathrm{ad}}, \gamma_{\mathrm{p}, \mathrm{max}}\right), \\
5.8 \times 10^{-5} \eta \nu_{\mathrm{obs}, \mathrm{keV}} E_{\mathrm{cur}, \mathrm{GeV}}^{-1} \\
\times \epsilon_{\mathrm{pc},-3} T_{\mathrm{pc}, 6.5}^{-1} B_{\mathrm{s}, 12}^{1 / 4} L_{\mathrm{sd}, 31}^{11 / 8} r_{6}^{-3 / 2} \\
\left(t_{\mathrm{cool}, \mathrm{syn}}>t_{\mathrm{ad}}, \gamma_{\mathrm{p}, \mathrm{E}}\right), \\
2.1 \times 10^{-4} \eta \nu_{\mathrm{obs}, \mathrm{keV}} \\
\times \epsilon_{\mathrm{pc},-3} T_{\mathrm{pc}, 6.5}^{-1} B_{\mathrm{s}, 12}^{1 / 2} L_{\mathrm{sd}, 31}^{-1 / 4} r_{6}^{-1} \\
\left(t_{\mathrm{cool}, \mathrm{syn}}>t_{\mathrm{ad}}, \gamma_{\mathrm{p}, \max }\right) .
\end{array}\right.
$$

The dependence of the efficiency $\epsilon_{\mathrm{syn}}$ in $\gamma \gamma$ scenario on the radius $r$ is shown in the upper panels of figure 2 Although we only show the case $\gamma_{\mathrm{p}}=\gamma_{\mathrm{p} \text {, } \mathrm{E}}$ in the upper panels of figure 2 the following trends are the same in the case $\gamma_{\mathrm{p}}=\gamma_{\mathrm{p}, \max }$. Under condition $t_{\mathrm{cool}, \mathrm{syn}}<t_{\mathrm{ad}}$ $\left(r<r_{\text {eq,syn }}\right)$, the efficiency $\epsilon_{\mathrm{syn}}$ increases toward the outer region (panels A and B in figure 2). Since the pitch angle $\alpha$ and the energy ratio $\gamma_{\mathrm{s}, \mathrm{syn}} / \gamma_{\mathrm{s} \text {,pair }}$ increase toward the outer region, the efficiency $\epsilon_{\mathrm{syn}}$ tends to increase with the increase of $r$. In the outer region $r>r_{\text {eq.syn }}$ $\left(t_{\mathrm{cool}, \mathrm{syn}}>t_{\mathrm{ad}}\right)$, the fraction of the total momentum of secondary particles, which loses via synchrotron radiation, decreases toward the large distance $r$. Thus, the efficiency $\epsilon_{\text {syn }}$ decreases toward the large distance $r$ (panels $\mathrm{B}$ and $\mathrm{C}$ in figure 2).

In a similar way as in equation (34), the efficiencies in $\mathrm{B} \gamma$ pair creation scenario are given by

$$
\epsilon_{\mathrm{syn}} \sim\left\{\begin{array}{c}
2.7 \times 10^{-4} \eta \nu_{\mathrm{obs}, \mathrm{keV}}^{1 / 2} \\
\times B_{\mathrm{s}, 12}^{1 / 8} L_{\mathrm{sd}, 31}^{3 / 1} r_{6}^{-3 / 4} \\
\left(t_{\mathrm{cool}, \mathrm{syn}}<t_{\mathrm{ad}}, \text { dipole }\right) \\
1.5 \times 10^{-1} \alpha_{0}^{3 / 2} \eta \zeta_{\mathrm{B}}^{1 / 2} \\
\times \nu_{\mathrm{obs}, \mathrm{keV}}^{1 / 2} B_{\mathrm{s}, 12}^{1 / 2} r_{6}^{-3 / 2} \\
\left(t_{\mathrm{cool}, \mathrm{syn}}<t_{\mathrm{ad}}, \text { non - dipole }\right) .
\end{array}\right.
$$

Schematic pictures for the efficiency in $\mathrm{B} \gamma$ scenario as a function of radius $r$ is shown in the lower panels of figure 2. As already mentioned, we only consider $t_{\mathrm{cool}, \mathrm{syn}}<t_{\mathrm{ad}}$ in $\mathrm{B} \gamma$ scenario (equation [30). The dependence of the efficiency $\epsilon_{\mathrm{syn}}$ on $r$ mainly comes from the photon escaping energy $E_{\text {esc }}$ (equation 15). Since the strong magnetic field makes the pair conversion efficient, the energy $E_{\text {esc }}$ decreases toward the neutron star surface. Then, the efficiency $\epsilon_{\text {syn }}$ increases toward the inner region (panels D and $\mathrm{E}$ in figure 2).

\subsection{Allowed Emission Region and Death Lines}

In order to derive the maximum luminosity of the synchrotron radiation in the allowed emission region of the magnetosphere, we have to consider the constraints on the emission region discussed in section 2.3. In this subsection, using the typical values, we derive the limits on 
YY scenario, $\gamma_{\mathrm{p}, \mathrm{E}}$, dipole

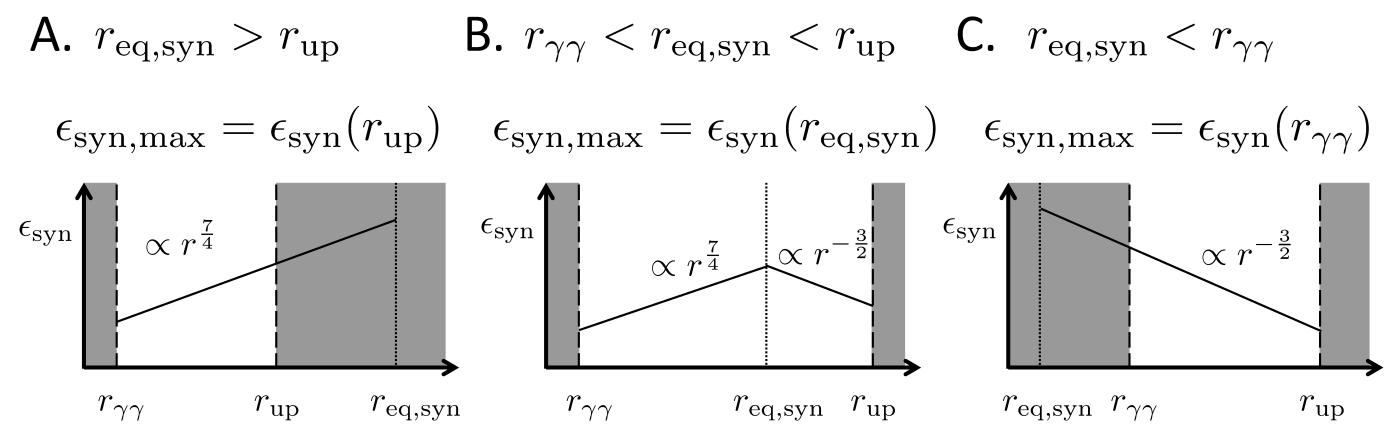

By scenario, $\boldsymbol{V}_{\mathrm{p} \text {, max }}$, dipole

D. $r_{\text {eq,syn }}>r_{\text {up }}$

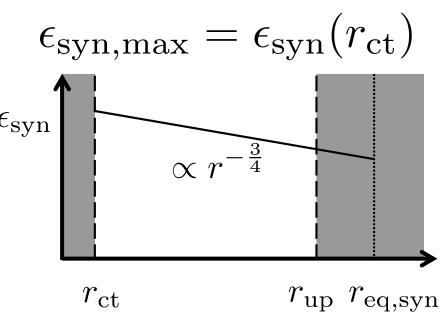

By scenario, $\boldsymbol{\gamma}_{\mathrm{p}, \max }$, non-dipole

E. $r_{\text {eq, syn }}>r_{\text {up }}$

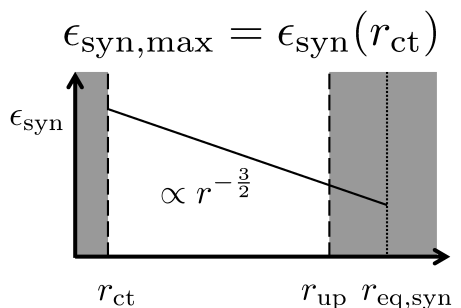

FIG. 2.- Schematic pictures for the $r$ dependence of the efficiency of the synchrotron radiation $\epsilon_{\mathrm{s} y n}$. The upper limits on the emission region are $r_{\mathrm{up}} \equiv \min \left\{R_{\mathrm{lc}}, r_{\gamma, \mathrm{syn}}\right\}$ for $\gamma \gamma$ scenario (panels $\mathrm{A}-\mathrm{C}$ ), and $r_{\mathrm{up}} \equiv \min \left\{R_{\mathrm{lc}}, r_{\mathrm{B} \gamma}\right\}$ for B $\gamma$ scenario (panels $\mathrm{D}$ and $\mathrm{E}$ ).

the emission region $r$ from the constraints (inequalities 20, 25]). From the energy condition for the secondary particles (21) and the B $\gamma$ pair creation threshold (25), the upper limits on the emission region $r$ are given by

$$
r_{\gamma \mathrm{syn}, 6} \sim\left\{\begin{array}{c}
87 \nu_{\mathrm{obs}, \mathrm{keV}}^{-2 / 5} E_{\mathrm{cur}, \mathrm{GeV}}^{4 / 5} B_{\mathrm{s}, 12}^{3 / 10} L_{\mathrm{sd}, 31}^{1 / 20}, \\
\left(\gamma_{\mathrm{p}, \mathrm{E}}, \text { dipole }\right), \\
12 \nu_{\mathrm{obs}, \mathrm{keV}}^{-2 / 7} B_{\mathrm{s}, 12}^{1 / 14} L_{\mathrm{sd}, 31}^{27 / 28}, \\
\left(\gamma_{\mathrm{p}, \max }, \text { dipole }\right), \\
71 \alpha_{0}^{1 / 5} \zeta_{\mathrm{B}}^{1 / 5} \nu_{\mathrm{obs}, \mathrm{keV}}^{1 / 5} B_{\mathrm{s}, 12}^{1 / 5} L_{\mathrm{sd}, 31}^{3 / 5}, \\
\left(\gamma_{\mathrm{p}, \mathrm{max}}, \text { non }- \text { dipole }\right),
\end{array}\right.
$$

and

$$
r_{\mathrm{B} \gamma, 6} \sim\left\{\begin{array}{c}
1.1 B_{\mathrm{s}, 12}^{1 / 6} L_{\mathrm{sd}, 31}^{7 / 12} \\
(\text { dipole }) \\
9.0 \alpha_{0}^{1 / 4} \zeta_{\mathrm{B}}^{1 / 4} B_{\mathrm{s}, 12}^{1 / 4} L_{\mathrm{sd}, 31}^{3 / 8} \\
(\text { non }- \text { dipole })
\end{array}\right.
$$

respectively. On the other hand, the lower limits are given by the conditions of the synchrotron approximation (23) and the $\gamma \gamma$ pair creation threshold (24),

$$
r_{\mathrm{ct}, 6} \sim\left\{\begin{array}{c}
6.7 \nu_{\mathrm{obs}, \mathrm{keV}}^{-2 / 7} B_{\mathrm{s}, 12}^{5 / 14} L_{\mathrm{sd}, 31}^{-1 / 28} \\
(\text { dipole }), \\
2.3 \alpha_{0}^{-1 / 3} \zeta_{\mathrm{B}}^{1 / 3} \nu_{\mathrm{obs}, \mathrm{keV}}^{-1 / 3} B_{\mathrm{s}, 12}^{1 / 3} \\
(\text { non }- \text { dipole }),
\end{array}\right.
$$

and

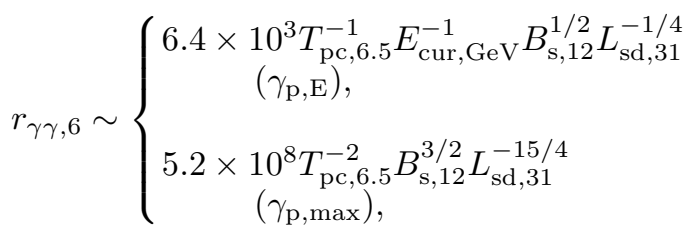

respectively.

In figure 3, we show the allowed range of the emission regions of the synchrotron radiation as the regions surrounded by solid lines as functions of the surface dipole field $B_{\mathrm{s}}$ and the spin-down luminosity $L_{\mathrm{sd}}$. We also show the contour lines of the efficiency $\epsilon_{\mathrm{syn}}$ with $\eta=1$ as dashed lines in the allowed emission region from equations (34) and (35).

In $\gamma \gamma$ pair creation scenario (left panels in figure 31), we fix the characteristic energy of the curvature photons $E_{\text {cur }}=3 \mathrm{GeV}$. The upper limit on the emission region $r$ is determined by the radius of the light cylinder $R_{\mathrm{lc}}$ (equation 11) for $h \nu_{\mathrm{obs}}=1 \mathrm{keV}$ (upper panel) and $1 \mathrm{eV}$ (lower panel), except for some low spin-down pulsars whose upper limit on the emission region is $r_{\gamma \mathrm{syn}}$ (equation 36) for $h \nu_{\mathrm{obs}}=1 \mathrm{keV}$. The lower limit is determined by the pair creation condition, $r_{\gamma \gamma}$ (equation 39). In X-ray band $\left(h \nu_{\mathrm{obs}}=1 \mathrm{keV}\right)$ the efficiency of the synchrotron radiation $\epsilon_{\text {syn }}$ becomes the maximum at the light cylinder $r=R_{\mathrm{lc}}$ (upper left panel in figure 3). For the range of the spin-down luminosity $L_{\mathrm{sd}} \lesssim 10^{34}-10^{35}$ erg $\mathrm{s}^{-1}$, there is no allowed emission region in the range $B_{\mathrm{s}} \gtrsim 10^{11} \mathrm{G}$. In optical band $\left(h \nu_{\mathrm{obs}}=1 \mathrm{eV}\right)$, the radius $r_{\text {eq,syn }}\left(t_{\text {cool,syn }}=t_{\text {ad }}\right.$; equation 30) is within the 


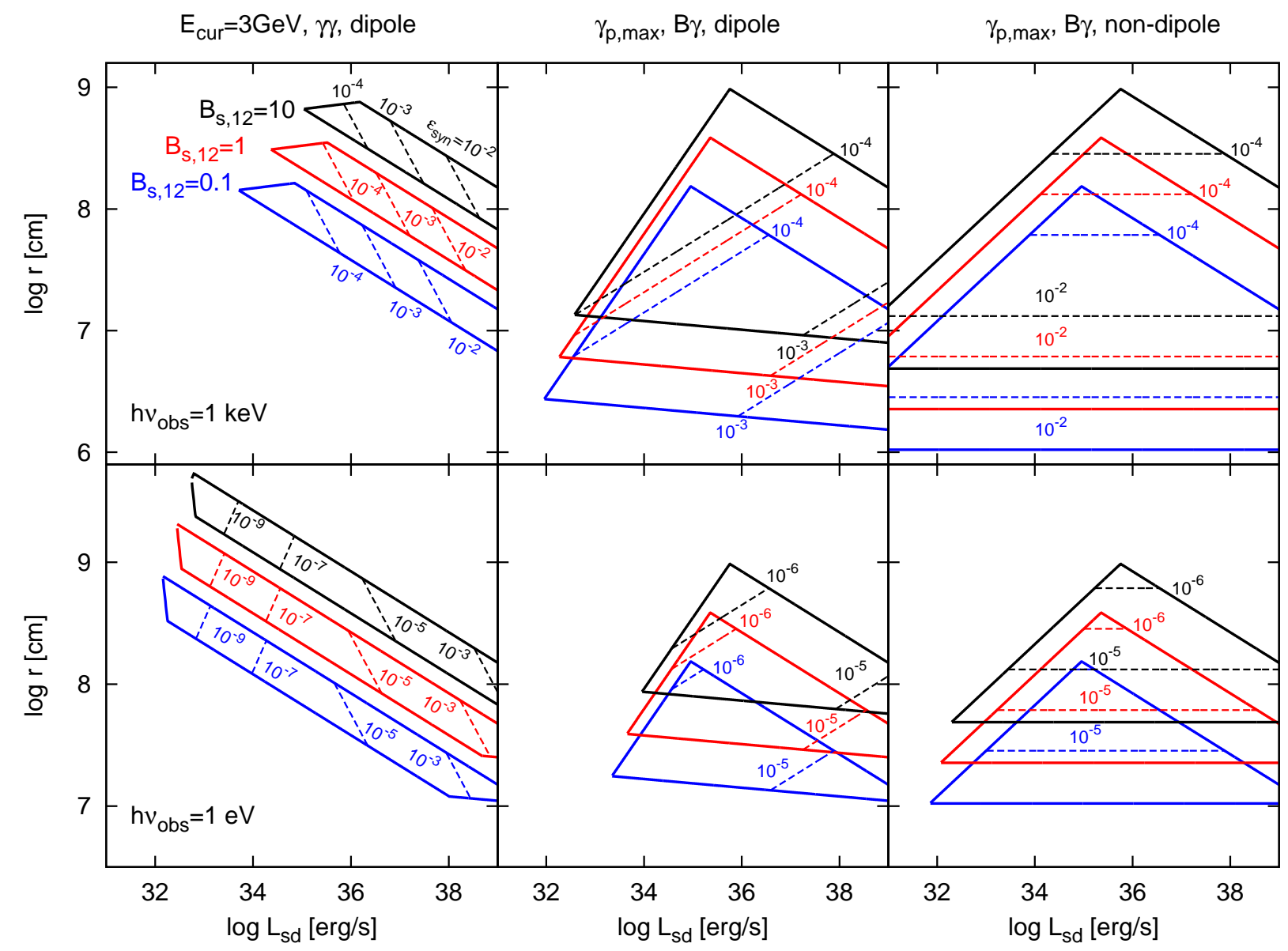

FIG. 3. - The allowed ranges of the emission region $r$ as functions of the spin-down luminosity $L_{\mathrm{sd}}$ for the surface dipole field $B_{\mathrm{S}}=10^{11} \mathrm{G}$ (blue), $10^{12} \mathrm{G}$ (red), and $10^{13} \mathrm{G}$ (black). The allowed ranges are surrounded by solid lines. Upper panels show the X-ray emission region $\left(h \nu_{\mathrm{obs}}=1 \mathrm{keV}\right)$, and lower panels show the optical emission region $\left(h \nu_{\mathrm{obs}}=1 \mathrm{eV}\right)$. The results in the scenarios of $\gamma \gamma$ with $E_{\mathrm{cur}}=3 \mathrm{GeV}$, $\mathrm{B} \gamma$ with dipole field, and $\mathrm{B} \gamma$ with non-dipole field are shown in left, middle, and right panels, respectively. The dashed lines denote the efficiency of the synchrotron radiation $\epsilon_{\mathrm{syn}}$ from equations (34) and (35). The parameters are fixed to $\alpha_{0}=\eta=\zeta_{\mathrm{B}}=1$.

allowed region for the pulsars with $L_{\text {sd }} \sim 10^{34}-10^{36} \mathrm{erg}$ $\mathrm{s}^{-1}$ (panel $\mathrm{B}$ in figure 2). The narrowness of the allowed emission region for a given $L_{\text {sd }}$ makes the range of the state in panel B very narrow. For pulsars with low spindown luminosity $L_{\text {sd }} \lesssim 10^{34} \mathrm{erg} \mathrm{s}^{-1}$, the radius $r_{\text {eq,syn }}$ is smaller than the lower limit on the emission region $r_{\gamma \gamma}$ (panel $\mathrm{C}$ of figure 2). Then, the maximum efficiency $\epsilon_{\mathrm{syn}}$ is given at the region $r=r_{\gamma \gamma}$ (lower left panel in figure 3). In fact, the slope of the contours in the lower left panel in figure 3 is opposite for $L_{\text {sd }} \lesssim 10^{34} \mathrm{erg} \mathrm{s}^{-1}$ and $L_{\text {sd }} \gtrsim 10^{36} \mathrm{erg} \mathrm{s}^{-1}$. Pulsars with $\widetilde{L}_{\mathrm{sd}} \lesssim 10^{32} \mathrm{erg} \mathrm{s}^{-1}$ cannot emit the synchrotron radiation at optical band from the secondary particles in our model.

In $\mathrm{B} \gamma$ pair creation scenario (middle and right panels in figure 3), the upper limit on the emission region is determined by the pair creation condition $r_{\mathrm{B} \gamma}$ for pulsars with $L_{\mathrm{sd}} \lesssim 10^{35}-10^{36}$ erg s$^{-1}$ or the light cylinder $R_{\mathrm{lc}}$ for pulsars with $L_{\mathrm{sd}} \gtrsim 10^{35}-10^{36} \mathrm{erg} \mathrm{s}^{-1}$. Since we use the maximum value $\gamma_{\mathrm{p} \text {,max }}$ as the Lorentz factor of the primary particles, the upper limit $r_{\mathrm{B} \gamma}$ is large and the allowed region is very broad compared with $\gamma \gamma$ scenario. The lower limit on the emission region is $r_{\text {ct }}$ derived by the validity condition of the synchrotron approximation (equation 23). Since the upper limits $r_{\mathrm{B} \gamma}$ and $R_{\mathrm{lc}}$ do not depend on the observed frequency $\nu_{\mathrm{obs}}$, the dependence of the area of the allowed region on the frequency $\nu_{\text {obs }}$ comes from the lower limit $r_{\text {ct }}$ (equation 38). In the non-dipole field dominated case at the emission region (right panels in figure 3), we fix the model parameters $\alpha_{0}=\zeta_{B}=1$. The allowed area is larger than that in the dipole case (middle panels in figure [3). The main difference between dipole and non-dipole dominated cases is the pitch angle $\alpha$ which could be an order of unity in non-dipole dominated case, while the pitch angle near the surface is typically an order of $\alpha \sim 10^{-2}$ in the dipole case (equation 6). The efficiency of the synchrotron radiation becomes the maximum value at the lower limit $r=r_{\text {ct }}$ for both dipole and non-dipole cases. In the $\mathrm{X}$ ray band $\left(h \nu_{\mathrm{obs}}=1 \mathrm{keV}\right)$, if the surface dipole field is $B_{\mathrm{s}} \lesssim 10^{11} \mathrm{G}$, the lower limit on the emission region is determined by the stellar radius $R_{\mathrm{ns}}$ (upper right panel in figure 3). In the optical band $\left(h \nu_{\text {obs }}=1 \mathrm{eV}\right)$, the lower limit $r_{\mathrm{ct}}$ reaches to the light cylinder radius $R_{\mathrm{lc}}$ for pulsars with very high spin-down luminosity $\left(L_{\mathrm{sd}} \gtrsim 10^{40} \mathrm{erg}\right.$ $\left.\mathrm{s}^{-1}\right)$. Then, the optical synchrotron radiation from the magnetosphere is not expected for such energetic pulsars irrespective of the existence of the non-dipole component.

In figure 4, the death lines in $\gamma \gamma$ scenario are shown 


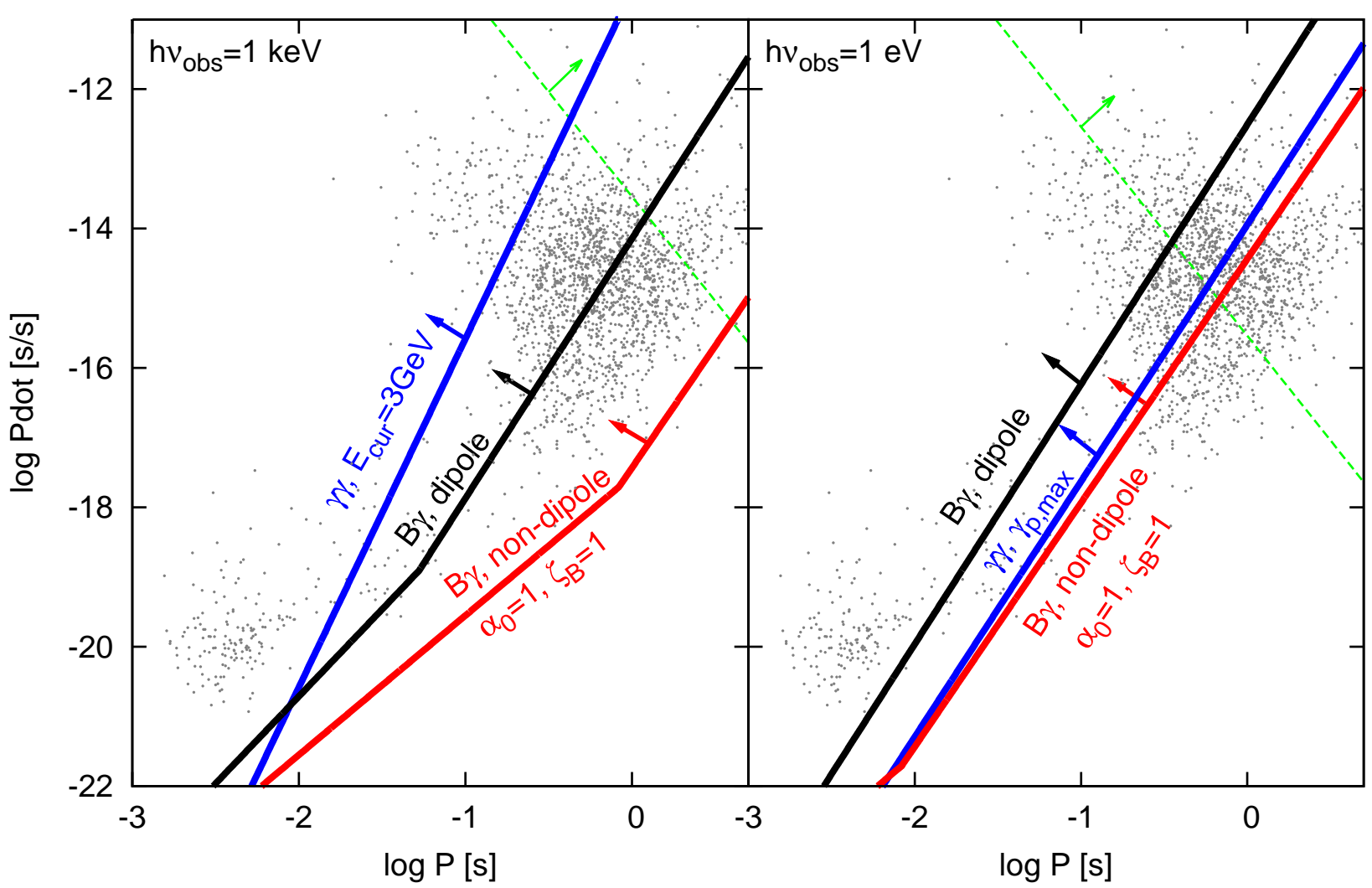

FIG. 4.- Death lines for the synchrotron radiation with $h \nu_{\mathrm{obs}}=1 \mathrm{keV}$ (left panel), and $1 \mathrm{eV}$ (right panel) on the $P$ - $\dot{P}$ diagram. Blue, black, and red solid lines denote the death lines from equations (40) and (42) for $\gamma \gamma$ scenario with $E_{\text {cur }}=3 \mathrm{GeV}, \mathrm{B} \gamma$ scenario with dipole field, and $\mathrm{B} \gamma$ scenario with non-dipole field $\left(\alpha_{0}=\zeta_{B}=1\right)$, respectively. Green dashed lines denote the steady emission conditions with $\epsilon_{\mathrm{B}}=10^{-3}$ (left) and $10^{-5}$ (right) from equation (48). Small dots denote pulsars taken from ATNF Pulsar Catalog (Manchester et al. 2005). Note that in optical case, the death lines for $\gamma \gamma$ scenario are determined by $\gamma_{\mathrm{p}, \text { max }}$ even if $E_{\mathrm{cur}}=3 \mathrm{GeV}$.

as blue lines on $P-\dot{P}$ diagram. We define the death line as the upper limit on the emission region is smaller than the lower limit. For pulsars which locate below the death lines in $P-\dot{P}$ diagram, there is no allowed region for synchrotron radiation from created secondary pairs in their magnetosphere. Using the equations (36) and (39), we derive the death lines for the synchrotron radiation from the pulsar magnetosphere in $\gamma \gamma$ scenario,

$$
\dot{P} \gtrsim\left\{\begin{array}{c}
5.2 \times 10^{-7} \nu_{\mathrm{obs}, \mathrm{keV}}^{2} E_{\mathrm{cur}, \mathrm{GeV}}^{-9} T_{\mathrm{pc}, 6.5}^{-5} P_{0}^{5} \mathrm{~s} \mathrm{~s}^{-1} \\
\left(r_{\gamma \gamma}<r_{\gamma \mathrm{syn}}, \gamma_{\mathrm{p}, \mathrm{E}}\right), \\
1.9 \times 10^{-14} \nu_{\mathrm{obs}, \mathrm{keV}}^{1 / 14} T_{\mathrm{pc}, 6.5}^{-1 / 2} P_{0}^{26 / 7} \mathrm{~s} \mathrm{~s}^{-1} \\
\left(r_{\gamma \gamma}<r_{\gamma \mathrm{syn}}, \gamma_{\mathrm{p}, \mathrm{max}}\right), \\
1.1 \times 10^{-14} T_{\mathrm{pc}, 6.5}^{-2 / 3} P_{0}^{11 / 3} \mathrm{~s} \mathrm{~s}^{-1} \\
\left(r_{\gamma \gamma}<R_{\mathrm{lc}}, \gamma_{\mathrm{p}, \max }\right) .
\end{array}\right.
$$

Condition $r_{\gamma \gamma}<R_{\mathrm{lc}}$ in $\gamma \gamma$ scenario with the Lorentz factor of primary particles $\gamma_{\mathrm{p}, \mathrm{E}}$ does not depend on the radius $r$. This condition corresponds to the inequality,

$$
E_{\mathrm{cur}, \mathrm{GeV}} T_{\mathrm{pc}, 6.5} \gtrsim 1.4 \quad\left(r_{\gamma \gamma}<R_{\mathrm{lc}}, \gamma_{\mathrm{p}, \mathrm{E}}\right),
$$

which also does not depend on $P$ and $\dot{P}$. From condition (41), since the observed temperature is typically $T_{\mathrm{Dc}} \sim 10^{6}-10^{6.5} \mathrm{~K}$ (e.g., Halpern \& Ruderman 1993; Becker 2009), the characteristic energy $E_{\text {cur }} \gtrsim 1-3 \mathrm{GeV}$ is required in our model. In $\gamma \gamma$ scenario with the Lorentz factor $\gamma_{\mathrm{p}, \mathrm{E}}$, the lower limit on the period derivative $\dot{P}$ depends on $\nu_{\text {obs }}^{2}$ from inequality (40). Then, the allowed parameter area in the $P-\dot{P}$ diagram becomes large for the low frequency $\nu_{\text {obs }}$. However, since the Lorentz factor $\gamma_{\mathrm{p}, \mathrm{E}}$ cannot exceed $\gamma_{\mathrm{p}, \max }$, we have to use $\gamma_{\mathrm{p} \text {,max }}$ to derive the death line in the case $\gamma_{\mathrm{p}, \mathrm{E}}>\gamma_{\mathrm{p} \text {,max }}$. In the $\mathrm{X}$ ray band, we consider the death line with $\gamma_{\mathrm{p}, \mathrm{E}}<\gamma_{\mathrm{p} \text {,max }}$ in most region on $P-\dot{P}$ diagram while in the optical band, we should consider the case $\gamma_{\mathrm{p}, \mathrm{E}}>\gamma_{\mathrm{p}, \max }$ for the death line. In the latter case, the death line is almost insensitive to $\nu_{\text {obs }}$ (inequality 40). Therefore, we do not expect any synchrotron radiation with frequency $h \nu_{\text {obs }} \lesssim 1 \mathrm{eV}$ from pulsars which reside below the death line with $\gamma_{\mathrm{p}, \max }$ in $\gamma \gamma$ scenario.

In $\mathrm{B} \gamma$ scenario, the death lines for the synchrotron ra- 
diation are derived from equations (37) and (38) as,

$$
\dot{P} \gtrsim\left\{\begin{array}{c}
7.2 \times 10^{-15} \nu_{\mathrm{obs}, \mathrm{keV}}^{-6 / 11} P_{0}^{41 / 11} \mathrm{~s} \mathrm{~s}^{-1} \\
\left(r_{\mathrm{ct}}<r_{\mathrm{B} \gamma}, \text { dipole }\right), \\
1.9 \times 10^{-16} P_{0}^{5 / 2} \mathrm{~s} \mathrm{~s}^{-1} \\
\left(R_{\mathrm{ns}}<r_{\mathrm{B} \gamma}, \text { dipole }\right), \\
3.7 \times 10^{-18} \alpha_{0}^{-7 / 4} \zeta_{\mathrm{B}}^{1 / 4} \nu_{\mathrm{obs}, \mathrm{keV}}^{-1} P_{0}^{7 / 2} \mathrm{~s} \mathrm{~s}^{-1} \\
\left(r_{\mathrm{ct}}<r_{\mathrm{B} \gamma}, \text { non }- \text { dipole }\right), \\
2.8 \times 10^{-18} \alpha_{0}^{-1 / 2} \zeta_{\mathrm{B}}^{-1 / 2} P_{0}^{2} \mathrm{~s} \mathrm{~s}^{-1} \\
\left(R_{\mathrm{ns}}<r_{\mathrm{B} \gamma}, \text { non }- \text { dipole }\right) .
\end{array}\right.
$$

We also show these death lines as black (dipole) and red lines (non-dipole) in figure 4. The dependence on the observed frequency $\nu_{\mathrm{obs}}$ mainly comes from the condition of the validity of the synchrotron approximation (inequality 23). From the left panel of figure 4, we expect that the synchrotron radiation with the frequency $h \nu_{\text {obs }}>1$ $\mathrm{keV}$ is emitted from the magnetosphere of almost all detected radio pulsars if the non-dipole component of the magnetic field dominates at the emission region. On the other hand, in the optical band $\left(h \nu_{\mathrm{obs}} \sim 1 \mathrm{eV}\right)$, we do not expect the emission of the synchrotron radiation from pulsars with $L_{\text {sd }} \lesssim 10^{32} \mathrm{erg} \mathrm{s}^{-1}$. Even if the non-dipole component enhances the magnetic field at the emission region $\left(\zeta_{\mathrm{B}}>1\right)$, the area of the allowed region with larger $P\left(P \gtrsim 1 \mathrm{~s}\right.$ for $\mathrm{X}$-ray, and $P \gtrsim 10^{-2} \mathrm{~s}$ for optical $)$ on the $P-\dot{P}$ diagram decreases because the lower limit $r_{\mathrm{ct}}$ increases.

\subsection{Maximum Luminosity of Synchrotron Radiation}

Using equations (34) and (35), and taking into account four constraints on the emission region (36]-39), we calculate the maximum luminosity of synchrotron radiation as a function of model parameters $\eta, \alpha_{0}$ and $\zeta_{\mathrm{B}}$. In $\gamma \gamma$ scenario, the synchrotron luminosity becomes maximum at $r=R_{\mathrm{lc}}$ (equation 1) or $r_{\gamma \mathrm{syn}}$ (equation 36), $r=r_{\text {eq,syn }}$ (equation 30), and $r=r_{\gamma \gamma}$ (equation 39) for panels A, B and C in figure 2, respectively. Substituting these radius into equation (34), we obtain the maximum luminosity. For the low- $L_{\text {sd }}$ pulsars which satisfy condition $t_{\mathrm{cool}, \mathrm{syn}}>t_{\mathrm{ad}}$ at the emission region (pane C), Lorentz factor of the primary particles could become $\min \left\{\gamma_{\mathrm{p}, \max }, \gamma_{\mathrm{p}, \mathrm{E}}\right\}=\gamma_{\mathrm{p}, \max }$. We use $\gamma_{\mathrm{p}, \max }$ only for the the condition in panel $\mathrm{C}$. The synchrotron luminosities are described as,

$$
L_{\mathrm{syn}} \lesssim\left\{\begin{array}{c}
4.6 \times 10^{23} \eta \nu_{\mathrm{obs}, \mathrm{keV}}^{1 / 2} E_{\mathrm{cur}, \mathrm{GeV}}^{-1} \\
\times \epsilon_{\mathrm{pc},-3} T_{\mathrm{pc}, 6.5}^{-1} B_{\mathrm{s}, 12}^{-1 / 4} L_{\mathrm{sd}, 31}^{15 / 8} \\
\quad\left(A, r_{\gamma \mathrm{syn}}>R_{\mathrm{lc}}, \gamma_{\mathrm{p}, \mathrm{E}}\right) \\
4.1 \times 10^{20} \eta \nu_{\mathrm{obs}, \mathrm{keV}}^{-1 / 5} E_{\mathrm{cur}, \mathrm{GeV}}^{2 / 5} \\
\times \epsilon_{\mathrm{pc},-3} T_{\mathrm{pc}, 6.5}^{-1} B_{\mathrm{s}, 12}^{-3 / 5} L_{\mathrm{sd}, 31}^{12 / 5} \\
\left(A, r_{\gamma \mathrm{syn}}<R_{\mathrm{lc}}, \gamma_{\mathrm{p}, \mathrm{E}}\right) \\
2.3 \times 10^{22} \eta \nu_{\mathrm{obs}, \mathrm{keV}}^{10 / 13} E_{\mathrm{cur}, \mathrm{GeV}}^{-1}{ }^{-1} \\
\times \epsilon_{\mathrm{pc},-3} T_{\mathrm{pc}, 6.5}^{-1} B_{\mathrm{s}, 12}^{-5 / 13} L_{\mathrm{sd}, 31}^{61 / 26} \mathrm{erg} \mathrm{s}^{-1} \\
\left(B, \gamma_{\mathrm{p}, \mathrm{E}}\right) \\
\times 10^{21} \eta \nu_{\mathrm{obs}, \mathrm{keV}} E_{\mathrm{cur}, \mathrm{GeV}}^{1 / 2} \\
\times \epsilon_{\mathrm{pc},-3} T_{\mathrm{pc}, 6.5}^{1 / 2} B_{\mathrm{s}, 12}^{-1 / 2} L_{\mathrm{sd}, 31}^{11 / 4} \mathrm{erg} \mathrm{s}^{-1} \\
\left(C, \gamma_{\mathrm{p}, \mathrm{E}}\right) \\
\times 10^{18} \eta \nu_{\mathrm{obs}, \mathrm{keV}} \\
\times \epsilon_{\mathrm{pc},-3} T_{\mathrm{pc}, 6.5} B_{\mathrm{s}, 12}^{-1} L_{\mathrm{sd}, 31}^{9 / 2} \mathrm{erg} \mathrm{s}^{-1} \\
\left(C, \gamma_{\mathrm{p}, \mathrm{max}}\right) .
\end{array}\right.
$$

In $\gamma \gamma$ scenario, a model parameter is only $\eta$. Note that if we extrapolate the derived luminosity to highly energetic pulsars $\left(L_{\mathrm{sd}} \gtrsim 10^{40} \mathrm{erg} \mathrm{s}^{-1}\right)$ in a simplistic form, the luminosity of the synchrotron radiation with $\eta=1$ seems to exceed the spin-down luminosity $L_{\mathrm{sd}}$. However, the optical depth $\tau_{\gamma \gamma}$ becomes higher than unity for such energetic pulsars. Then, the number flux of the created pair does not depend on the optical depth $\left(\min \left\{\tau_{\gamma \gamma}, 1\right\}=\right.$ 1 in equation [12). As a result, the luminosity of the synchrotron radiation follows the relation $L_{\mathrm{syn}} \propto L_{\mathrm{sd}}^{5 / 8}$, which always satisfies $L_{\text {syn }}<L_{\text {sd }}$ even if $\eta=1$.

On the other hand, the maximum luminosity of synchrotron radiation in $\mathrm{B} \gamma$ scenario are derived as,

$$
L_{\mathrm{syn}} \lesssim\left\{\begin{aligned}
6.4 \times 10^{26} \eta \nu_{\mathrm{obs}, \mathrm{keV}}^{5 / 7} \\
\times B_{\mathrm{s}, 12}^{-1 / 7} L_{\mathrm{sd}, 31}^{17 / 14} \mathrm{erg} \mathrm{s}^{-1} \\
(D, \text { dipole }), \\
4.5 \times 10^{29} \alpha_{0}^{2} \eta \nu_{\mathrm{obs}, \mathrm{keV}} L_{\mathrm{sd}, 31} \text { erg s}^{-1} \\
(E, \text { non }- \text { dipole }) .
\end{aligned}\right.
$$

The maximum luminosities are given at $r=r_{\mathrm{ct}}$. In the non-dipole case, the lower limit depends on the magnetic field, $r_{\mathrm{ct}} \propto \zeta_{\mathrm{B}}^{1 / 3}$ (equation 38 ). The dependence of the luminosity $L_{\mathrm{syn}}$ on the parameter $\zeta_{\mathrm{B}}$ and the emission region $r$ is $L_{\text {syn }} \propto \zeta_{\mathrm{B}}^{1 / 2} r^{-3 / 2}$ (equation [35). Then, the maximum luminosity in the non-dipole field dominant case does not depend on the parameter $\zeta_{\mathrm{B}}$.

In figure 5. we show the derived upper limits with $\alpha_{0}=$ $\eta=1$ on the $L_{\text {syn }}$ versus $L_{\text {sd }}$ planes. For the range of the surface dipole field, we consider $10^{11} \mathrm{G}<B_{\mathrm{s}}<10^{13} \mathrm{G}$. We plot the observed luminosities of the non-thermal X-ray $L_{\mathrm{X}}$ (left panel) and optical emissions $L_{\text {opt }}$ (right panel), and the spin-down luminosities $L_{\text {sd }}$. For the nonthermal optical emission, we also plot the observed upper limits.

In the X-ray band, the observed non-thermal luminosities of most pulsars with $L_{\mathrm{sd}} \gtrsim 10^{35} \mathrm{erg} \mathrm{s}^{-1}$ are compara- 

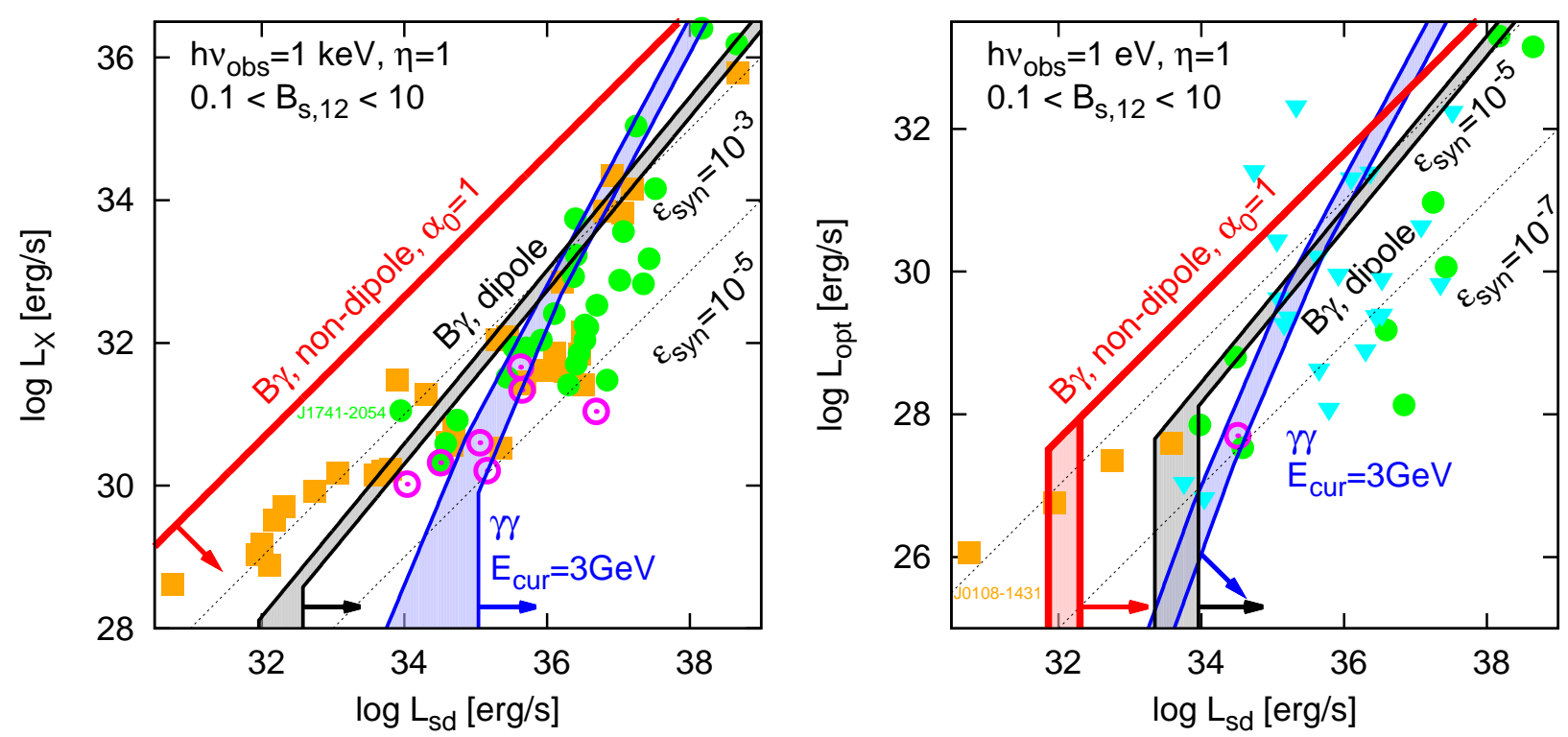

FIG. 5. - The maximum luminosities of synchrotron radiation in X-ray (left) and optical bands (right) for scenarios of $\gamma \gamma$ with $E_{\text {cur }}=3$ $\mathrm{GeV}$ (blue), $\mathrm{B} \gamma$ with dipole field (black), and $\mathrm{B} \gamma$ with non-dipole field (red) as a function of $L_{\mathrm{sd}}$. The observed luminosities in X-ray and optical bands are also plotted. Data are taken from Kargaltsev \& Pavlov (2008); Kargaltsev et al. (2012); Posselt et al. (2012b); Abdo et al. (2013); Prinz \& Becker (2015); Szarv et al. (2017) in X-ray, and from Zharikov \& Mignani] (2013); Beronva et al. (2015); Kirichenko et al. (2015); Mignani et al. (2016a b c); Shibanov et al. (2016) in optical. Filled green and open magenta circles are radioloud and radio-quiet $\gamma$-ray pulsars, respectively. Orange squares denote the non- $\gamma$-ray pulsars. In the right panel, upper limits on the optical luminosity are also plotted as cyan triangles. The parameters are fixed to $\alpha_{0}=\eta=\zeta_{\mathrm{B}}=1$. Note that the dependence of $\zeta_{\mathrm{B}}$ only appears to the lower boundary of $L_{\mathrm{sd}}$ in $\mathrm{B} \gamma$ scenario with non-dipole field.

ble to or lower than the maximum luminosity with $\eta=1$ in $\gamma \gamma$ pair creation scenario (equation 43, blue curve in the left panel of figure 5). The observed X-ray luminosities are $\sim 10^{-2}-1$ times lower than the maximum value. Then, the conversion efficiency should be $\eta \gtrsim 10^{-2}$. On the other hand, for pulsars with $L_{\mathrm{sd}} \lesssim 10^{35} \mathrm{erg} \mathrm{s}^{-1}$ including some $\gamma$-ray pulsars (green and magenta points), the observed non-thermal luminosities exceed the maximum luminosity in $\gamma \gamma$ scenario. These observed X-ray luminosities are explained in $\mathrm{B} \gamma$ scenario with dipole magnetic field (equation 44, black curve), except for PSR J1741-2054. For J1741-2054 and other pulsars with $L_{\mathrm{sd}} \lesssim 10^{34} \mathrm{erg} \mathrm{s}^{-1}$, we should take into account the nondipole component of the magnetic field at the emission region (equation 44 red line). Then, the observed luminosities for all samples are lower than the maximum value with $\eta=1$ and $\alpha_{0}=1$.

In the optical band, the right panel of figure 5 shows that the luminosity of the synchrotron radiation with $\eta=1$ in $\gamma \gamma$ scenario (equation 43, blue curve) is also higher than the observed non-thermal optical luminosities for pulsars with $L_{\mathrm{sd}} \gtrsim 10^{35} \mathrm{erg} \mathrm{s}^{-1}$. For pulsars with $10^{33} \mathrm{erg} \mathrm{s}^{-1} \lesssim L_{\text {sd }} \lesssim 10^{35} \mathrm{erg} \mathrm{s}^{-1}$, the observed optical luminosities are lower than the maximum luminosity with $\eta=1$ in $\mathrm{B} \gamma$ scenario with dipole magnetic field (equation 44; black curve). For pulsars with $10^{32}$ $\mathrm{erg} \mathrm{s}^{-1} \lesssim L_{\mathrm{sd}} \lesssim 10^{33} \mathrm{erg} \mathrm{s}^{-1}$, the non-dipole component are required for which the maximum luminosity with $\eta=1$ and $\alpha_{0}=1$ exceeds their observed nonthermal optical luminosities (equation 44, red curve).
For pulsars with $L_{\mathrm{sd}} \lesssim 10^{32} \mathrm{erg} \mathrm{s}^{-1}$, from condition $r_{\mathrm{ct}}<r_{\mathrm{B} \gamma}$ (equation 42), the synchrotron radiation in our model is not expected in optical band. The lower limit on the spin-down luminosity is proportional to the model parameter, $L_{\mathrm{sd}} \propto \zeta_{\mathrm{B}}^{1 / 4}$, derived from inequality (42), so that the strong magnetic field $\zeta_{\mathrm{B}}>1$ increases the lower limit on $L_{\text {sd }}$. PSR J0108-1431 with $L_{\mathrm{sd}} \sim 8 \times 10^{30} \mathrm{erg} \mathrm{s}^{-1}$ have been detected in optical band (Mignani. Pavlov \& Kargaltsev 2008). We will discuss this object in section 4 .

\subsection{Luminosity Ratio}

In our model, the fluxes of the curvature and the synchrotron radiations should be related. Then, the observed $\gamma$-ray, X-ray and optical emissions should also be related, unless the curvature photons are significantly absorbed in the magnetosphere. In $\gamma \gamma$ scenario, the absorption of the curvature photons is negligible for most $\gamma$-ray pulsars because of the low optical depth $\tau_{\gamma \gamma} \ll 1$. The luminosity of curvature radiation in our model is described by,

$$
L_{\text {cur }} \sim P_{\text {cur }} \dot{N}_{\mathrm{p}} \min \left\{t_{\text {cool,cur }}, t_{\text {ad }}\right\} .
$$

Within the typical range of the parameters for $\gamma$-ray pulsars, condition $t_{\text {cool,cur }}<t_{\text {ad }}$ is satisfied (equations 31 and 32). Using this condition and equations (16) and (17), the curvature luminosity $L_{\text {cur }}$ is derived as

$$
L_{\text {cur }} \sim \eta L_{\text {sd }} .
$$



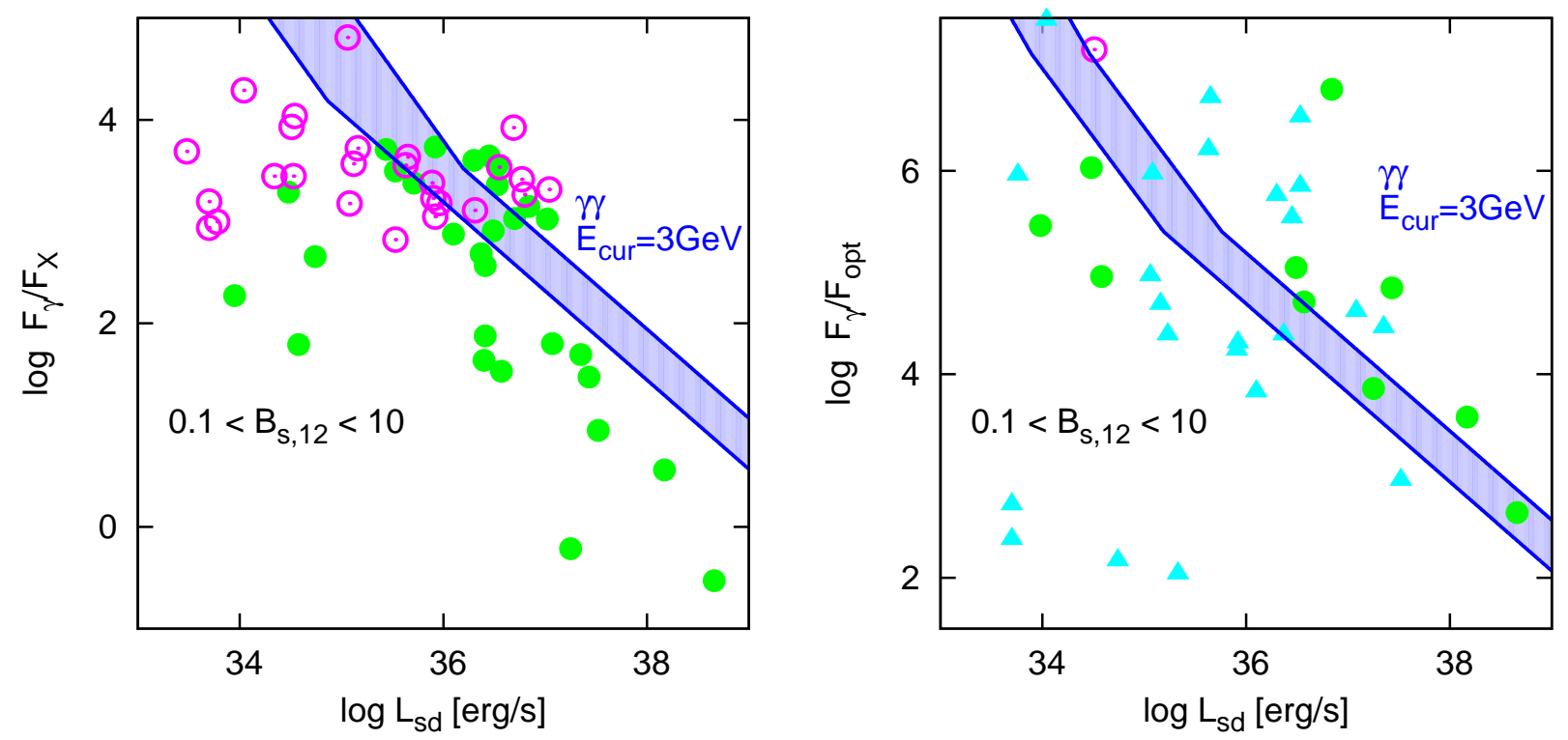

Fig. 6. - Flux ratios $F_{\gamma} / F_{\mathrm{X}}$ (left) and $F_{\gamma} / F_{\mathrm{opt}}$ (right) versus $L_{\mathrm{sd}}$ for $\gamma$-ray pulsars. The symbols are the same as in figure 5 Note that the cyan triangles are lower limits on $F_{\gamma} / F_{\text {opt }}$ in the right panel. Data are taken from Abdo et al. (2013); [Marelli et al. (2015); Beronva et al. (2015). Blue curves are the ratio $L_{\mathrm{cur}} / L_{\mathrm{syn}}$ in $\gamma \gamma$ scenario, which is calculated from equations (43) and (45).

From equation (34), the synchrotron efficiency is proportional to the conversion efficiency, $\epsilon_{\text {syn }} \propto \eta$. Then, the luminosity (or the flux) ratio $L_{\text {cur }} / L_{\text {syn }} \sim\left(\epsilon_{\text {syn }} / \eta\right)^{-1}$ does not depend on the conversion efficiency $\eta$.

In figure 6. we plot the observed $\gamma$-ray to X-ray (left panel) and $\gamma$-ray to optical (right panel) flux ratios for $\gamma$-ray pulsars. Since the flux ratios do not depend on the distance from us, we also plot the observed ratios for the radio-quiet $\gamma$-ray pulsars (magenta) whose distance are not well constrained. For the pulsars with $L_{\mathrm{sd}} \gtrsim 10^{36} \mathrm{erg}$ $\mathrm{s}^{-1}$, the observed flux ratios are an order of magnitude smaller than our model for X-ray band. As discussed in section 4.2 for energetic pulsars $\left(L_{\mathrm{sd}} \gtrsim 10^{36} \mathrm{erg} \mathrm{s}^{-1}\right)$, the thermal photons from entire surface which we do not consider in the model could contribute to the optical depth $\tau_{\gamma \gamma}$. The observed flux ratios of pulsars with $L_{\text {sd }} \lesssim 10^{35}$ erg $\mathrm{s}^{-1}$ are also much smaller than our model for both X-ray and optical bands. For these low- $L_{\text {sd }}$ pulsars, we should consider other effects such as the contribution of the synchrotron radiation from inner region in $\mathrm{B} \gamma$ scenario as discussed in section 4 . Note that in $\mathrm{B} \gamma$ scenario, significant curvature photons could be absorbed in the magnetosphere. Then, the observed $\gamma$-ray flux could be much smaller than X-ray and optical fluxes, so that we do not plot the flux ratio in $\mathrm{B} \gamma$ scenario in figure 6 .

\section{DISCUSSION}

In this paper, we analytically calculate the luminosity of the synchrotron radiation from the secondary particles created in the pulsar magnetosphere. In order to constrain the efficiency of the energy conversion from the loss rate of the rotation energy to the kinetic energy flux of the particles, we introduce a model parameter $\eta$ and compare with observations in X-ray and op- tical bands. X-ray and optical emissions are detected in not only young, high- $L_{\mathrm{sd}} \gamma$-ray pulsars $\left(\tau_{\mathrm{c}} \lesssim 10^{6} \mathrm{yr}\right.$, $\left.L_{\mathrm{sd}} \gtrsim 10^{33}-10^{34} \mathrm{erg} \mathrm{s}^{-1}\right)$, but also old, low- $L_{\mathrm{sd}}$ pulsars $\left(\tau_{\mathrm{c}} \gtrsim 10^{6} \mathrm{yr}, L_{\mathrm{sd}} \lesssim 10^{33}-10^{34} \mathrm{erg} \mathrm{s}^{-1}\right)$. We consider $\gamma \gamma$ and $\mathrm{B} \gamma$ pair creation processes as the electron/positron pair conversion process from the curvature photons emitted by the primary particles (figure 1). For the energy of primary particles, we use the observed value of the $\gamma$-ray spectral cutoff energy in $\gamma \gamma$ scenario. This model does not need to assume the strength of the electric field at the particle acceleration region, which is highly uncertain. In $\mathrm{B} \gamma$ scenario, we use the maximum value of the potential drop across the polar cap to obtain the energy of the primary particles, although the derived synchrotron luminosity does not depend on the Lorentz factor of the primary particles. The newly created secondary particles have a non-zero value of a pitch angle and emit the synchrotron radiation. For the magnetic field, we assume the dipole field in the magnetosphere. Near the stellar surface, we also include the effects of the non-dipole field on the pitch angle of the secondary particles and the curvature radius of the magnetic field line. In order to calculate the maximum synchrotron luminosity, we take into account the requirements for the radiation mechanism being synchrotron radiation.

In $\mathrm{B} \gamma$ scenario, the region where the luminosity of the synchrotron radiation becomes maximum is near the neutron star surface (figure 3). The synchrotron radiation from secondary pairs can be detected from pulsars with the spin-down luminosity $L_{\mathrm{sd}} \gtrsim 10^{32} \mathrm{erg} \mathrm{s}^{-1}$ for X-ray band and $L_{\mathrm{sd}} \gtrsim 10^{33}$ erg $\mathrm{s}^{-\widetilde{1}}$ for optical band in our $\mathrm{B} \gamma$ scenario with the dipole magnetic field (figure 4). The synchrotron efficiency for typical pulsars is $\epsilon_{\text {syn }} \sim\left(10^{-4}-10^{-3}\right) \nu_{\text {obs,keV }}^{5 / 7}$. The dependence on the 
spin-down luminosity is $L_{\text {syn }} \propto L_{\text {sd }}^{17 / 14}$ which is close to the linear trend. While the observed luminosities for high- $L_{\mathrm{sd}}$ pulsars $\left(L_{\mathrm{sd}} \gtrsim 10^{34} \mathrm{erg} \mathrm{s}^{-1}\right)$ could be explained by our model with dipole dominated case, the luminosities for low- $L_{\text {sd }}$ pulsars $\left(L_{\text {sd }} \lesssim 10^{34} \mathrm{erg} \mathrm{s}^{-1}\right)$ exceed the maximum synchrotron luminosity with $\eta=1$. In the non-dipole dominant case at the emission region, most pulsars reside the allowed area on the $P-\dot{P}$ diagram for X-ray band (figure 5). On the other hand, we expect that the synchrotron radiation is not detected from pulsars with $L_{\text {sd }} \lesssim 10^{32} \mathrm{erg} \mathrm{s}^{-1}$ in the optical band. The luminosity of the synchrotron radiation is $L_{\text {syn }} \sim 4 \times 10^{-2} \alpha_{0}^{2} \eta \nu_{\text {obs, keV }} L_{\text {sd }}$, which does not depend on the magnetic field strength at the emission region.

In $\gamma \gamma$ scenario (figure 5), the allowed region of the synchrotron radiation resides near the light cylinder (figure 3). The synchrotron death line in X-ray band corresponds to $L_{\mathrm{sd}} \sim 10^{34}-10^{35} \mathrm{erg} \mathrm{s}^{-1}$. In optical band, the synchrotron death line corresponds to $L_{\mathrm{sd}} \sim 10^{32}$ erg $\mathrm{s}^{-1}$, although the synchrotron efficiency becomes much small $\left(\epsilon_{\mathrm{syn}} \lesssim 10^{-7} \eta\right)$ for the range $L_{\mathrm{sd}} \lesssim 10^{34}$ $\operatorname{erg~s}{ }^{-1}$. The synchrotron luminosity dependence on the spin-down luminosity is $L_{\text {syn }} \propto L_{\text {sd }}^{a}$ with $a \sim 2$. In our model, the normalized thermal luminosity from the heated polar cap $\epsilon_{\mathrm{pc}}$ is constant based on the observations (Becker \& Trümper 1997; Becker 2009). Then, the number of thermal photons is proportional to the spin-down luminosity. The synchrotron luminosity is also proportional to the total energy of the primary particles, so that the index becomes $a \sim 2$. The observed non-thermal X-ray and optical emissions for $\gamma$-ray pulsars with $L_{\text {sd }} \gtrsim 10^{35} \mathrm{erg} \mathrm{s}^{-1}$ are lower than the maximum luminosity of our model with $\eta=1$ (figure 5). On the other hand, for the $\gamma$-ray pulsars with low spindown luminosity, $L_{\mathrm{sd}} \lesssim 10^{35} \mathrm{erg} \mathrm{s}^{-1}$, their observed luminosities exceed the maximum luminosity with $\eta=1$ in $\gamma \gamma$ scenario. Since $\gamma$-ray pulsars show no significant absorption feature via $\mathrm{B} \gamma$ process in their observed spectra (Abdo et al. 2013), the curvature radiation by the primary particles should be emitted from the outer magnetosphere. Then, in addition to the outer magnetosphere, another synchrotron emitting region may reside for $\gamma$-ray pulsars (see section 4.2).

Although our model only consider the region $r \leq R_{\mathrm{lc}}$, the derived synchrotron luminosity in $\gamma \gamma$ scenario with $r=R_{\mathrm{lc}}$ gives the maximum value for the cases where particle acceleration and high-energy emission take place in the current sheet outside the light cylinder. Recent numerical simulations indicate that a significant fraction of Poynting flux dissipates at $\sim 1-2 R_{\mathrm{lc}}$ (Belyaev 2015; Cerutti et al. 2015). Since the dissipation region is very close to the light cylinder $\left(\lesssim 2 R_{\mathrm{lc}}\right)$, the synchrotron efficiency would be comparable to the values in equation (34) with $r=R_{\mathrm{lc}}$. If our model extend outside the light cylinder, the maximum synchrotron efficiency could be given at $r=R_{\mathrm{lc}}$ as follows. The synchrotron efficiency in equation (28) is applicable to the region outside the light cylinder if the conditions for the timescales, $t_{\text {cool,syn }}<t_{\text {ad }}$ and $t_{\text {cool,cur }}<t_{\text {ad }}$, are satisfied. For simplicity, we assume $\alpha \sim 1$, $\cos \theta_{\text {col }} \sim 0$, and $R_{\text {cur }} \sim r$ at the region $r>R_{\mathrm{lc}}$. Since the toroidal component of the magnetic field would be dominant outside the light cylinder, we assume $B \propto r^{-1}$ at $r>R_{\mathrm{lc}}$. Then, the Lorentz factor of synchrotron emitting particles and the optical depth are $\gamma_{\mathrm{s}, \mathrm{syn}} \propto r^{1 / 2}$ (equation [10) and $\tau_{\gamma \gamma} \propto r^{-1}$ (equation [13), respectively. We also consider $\gamma_{\mathrm{p}, \mathrm{E}}<\gamma_{\mathrm{p} \text {, max }}$ for the Lorentz factor of the primary particles, so that the Lorentz factor $\gamma_{\mathrm{s} \text {,pair }}$ does not depend on $r$ (equation 22). Using equation (28), the synchrotron efficiency deceases as the large distance, $\epsilon_{\text {syn }} \propto r^{-1 / 2}$, and the efficiency becomes maximum at the light cylinder radius $r=R_{\mathrm{lc}}$. Therefore, $\gamma \gamma$ scenario with $r=R_{\mathrm{lc}}$ gives the upper limit on the efficiency $\epsilon_{\mathrm{syn}}$ even if most particle acceleration takes place outside the light cylinder.

Note that we use $\gamma_{p, E}$ as the Lorentz factor of primary particles. Then, the effective number flux of primary particle $\dot{N}_{\mathrm{p}}$ is $\eta \gamma_{\mathrm{p}, \max } / \gamma_{\mathrm{p}, \mathrm{E}}$ times larger than the GJ number flux, $2 \pi r_{\mathrm{pc}}^{2} \rho_{\mathrm{GJ}, \mathrm{sur}} c / e$ Goldreich \& Julian 1969 ), where $\rho_{\mathrm{GJ}, \text { sur }}$ is GJ charge density at the neutron star surface and $r_{\mathrm{pc}}$ is the polar cap radius. The primary particle is a carrier of current flowing the magnetosphere. It seems that the averaged current density exceeds the GJ value $\rho_{\mathrm{GJ}} c$. However, if the cooling timescale is smaller than the advection timescale, the primary particles are continuously accelerated and lose their energy via curvature radiation in the acceleration region. Then, the actual number of the particles could be $t_{\text {cool,cur }} / t_{\text {ad }}$ times smaller than the effective value. The minimum number of the primary particles normalized by the GJ value is $\eta\left(\gamma_{\mathrm{p}, \max } / \gamma_{\mathrm{p}, \mathrm{E}}\right)\left(t_{\text {cool,cur }} / t_{\mathrm{ad}}\right) \sim$ $1.1 \eta\left(E_{\text {cur }} / 3 \mathrm{GeV}\right)^{-4 / 3}\left(B_{\mathrm{s}} / 10^{12} \mathrm{G}\right)^{-1 / 6}\left(L_{\mathrm{sd}} / 10^{35} \mathrm{erg} \mathrm{s}^{-1}\right)^{7 / 12}$, which is an order of unity. Therefore, in our model, we do not consider that the current flowing the magnetosphere significantly exceeds the GJ current.

The synchrotron luminosities with $\eta=1$ (equations 43 and 44) provide the maximum luminosities which correspond to the theoretical critical lines on $L_{\mathrm{X}}$ versus $L_{\text {sd }}$ and $L_{\text {opt }}$ versus $L_{\text {sd }}$ planes. Using the observed non-thermal X-ray luminosity, Kargaltsev et al. (2012) found the critical lines which are described by broken power-law functions with the transition point $L_{\mathrm{sd}} \sim 10^{35}$ erg $\mathrm{s}^{-1}$. Their transition point is consistent with the point where the synchrotron luminosities of $\gamma \gamma$ and $\mathrm{B} \gamma$ scenarios are comparable in our model. For $L_{\mathrm{sd}} \geq 10^{35}$ erg s${ }^{-1}$, the critical line suggested by Kargaltsev et al. (2012) is roughly consistent with the maximum luminosity in $\gamma \gamma$ scenario. For $10^{33} \mathrm{erg} \mathrm{s}^{-1} \lesssim L_{\mathrm{sd}} \lesssim 10^{35} \mathrm{erg} \mathrm{s}^{-1}$, the index of their derived critical line is small, $L_{\mathrm{X}} \propto L_{\mathrm{sd}}^{0.38}$ (Kargaltsev et al. 2012). The small value of the index is possible if pulsars which have the non-dipole field at the emission region are preferentially detected at the range $L_{\text {sd }} \lesssim 10^{34}$ erg s$^{-1}$. For $L_{\text {sd }} \lesssim 10^{33}$ erg s $^{-1}$, we suggest that the critical line follows $L_{\text {syn }} \propto L_{\text {sd }}$ from $\mathrm{B} \gamma$ scenario with the non-dipole field. Our theoretical critical lines would be useful to select the observational target in optical and X-ray bands.

For PSR J0108-1431, the observed luminosity of the non-thermal X-ray emission is within the maximum value in our model, although the extreme conditions that the non-dipole field is dominant and the conversion efficiency $\eta$ and the pitch angle $\alpha_{0}$ are an order of unity are required. However, the non-thermal optical emission via synchrotron radiation is not expected from PSR J0108-1431 in our model. Even if we take into account the effect of the non-dipole component 
of the magnetic field, the synchrotron luminosity does not depend on the strength of the magnetic field $\zeta_{\mathrm{B}}$. Instead, the large value of $\zeta_{\mathrm{B}}(>1)$ makes the lower boundary of the spin-down luminosity high because the death line for condition $r_{\mathrm{ct}}<r_{\mathrm{B} \gamma}$ is $\dot{P} \propto \zeta_{\mathrm{B}}^{1 / 4}$ (equation 42). The significance of the optical detection of J0108-1431 is marginal (Mignani, Pavlov \& Kargaltsev 2008) and subsequent observation could not detect this pulsar (Mignani, Pavlov \& Kargaltsev 2011). If the non-thermal optical emission from J0108-1431 is confirmed, we should consider the other emission mechanisms and/or the other energy sources. Future observations will confirm whether the detected signal comes from J0108-1431 or not.

The region where the optical luminosity of the synchrotron radiation becomes maximum is $r \sim 10-50 R_{\mathrm{ns}}$ in $\mathrm{B} \gamma$ scenario (figure [3). This region is also considered as the coherent radio emission site from observations (e.g., Pilia et al. 2016). For low- $L_{\text {sd }}$ pulsars $\left(L_{\mathrm{sd}} \lesssim 10^{34} \mathrm{erg} \mathrm{\textrm {s } ^ { - 1 }}\right)$ which are required for the non-dipole component to explain their X-ray and optical luminosities, the radio pulse profile has high complexity (Karastergiou \& Johnston 2007) and the degree of linear polarization in radio is significantly lower than the high $L_{\text {sd }}$ pulsars (Weltevrede \& Johnston 2008). The modulation phenomena such as nulling and mode changing are also seen in the low- $L_{\text {sd }}$ pulsars (Wang, Manchester \& Johnston 2007). These facts support that the non-dipole magnetic field is a main control parameter for not only the X-ray and optical emission but also the radio emission for low- $L_{\text {sd }}$ pulsars.

\subsection{Energy Conversion Efficiency}

Comparing with observed values (figure [5), the conversion efficiency $\eta$ should be $\gtrsim 0.01-1$ in $\gamma \gamma$ scenario for pulsars with $L_{\text {sd }} \gtrsim 10^{35} \mathrm{erg} \mathrm{s}^{-1}$. In B $\gamma$ scenario with the dipole dominant case, the efficiency should be $\eta \gtrsim 0.01-1$ for pulsars with $L_{\text {sd }} \gtrsim 10^{34} \mathrm{erg} \mathrm{s}^{-1}$. Even if we consider the effects of the non-dipole component in B $\gamma$ scenario, the efficiency should be $\eta \gtrsim 10^{-4}-0.1$ with $\alpha_{0}=1$. We do not find any dependence of the efficiency $\eta$ on $P$ and $\dot{P}$. Note that our model has optimistic assumptions to enlarge the synchrotron luminosity as also discussed in Kisaka \& Tanaka (2014). Therefore, the actual efficiency $\eta$ would be larger than that derived from our model.

The efficiency parameter $\eta$ almost corresponds to $\sim(1+\sigma) / \sigma$, where $\sigma$ is the magnetization parameter (the ratio of the Poynting to the kinetic energy fluxes). Although the magnetization parameter is usually considered as $\sigma \gg 1$ in the magnetosphere (e.g., Daugherty \& Harding 1982), the requirement of $\eta \sim$ $O(0.1)(\sigma \sim O(10))$ for observations means that the significant electromagnetic energy have to convert to the particle energy in the magnetosphere. Especially, in B $\gamma$ scenario, the energy conversion to $\eta \sim O(0.1)$ should occur near the stellar surface.

In $\mathrm{B} \gamma$ scenario, the emission regions where the synchrotron luminosities in optical and X-ray become maximum are different (figure 3). If X-ray and optical emissions come from the same region in $\mathrm{B} \gamma$ scenario with non-dipole dominated case, X-ray luminosity is reduced by a factor of $\sim 30$ because of the frequency dependence of the lower limit on the emission region $r_{\mathrm{ct}} \propto \nu_{\mathrm{obs}}^{-1 / 3}$ (equation 38) and the distance dependence of the luminosity $L_{\text {syn }} \propto r^{-3 / 2}$ (equation 35). Then, the efficiency $\eta$ should be an order of unity at least for old pulsars which the non-dipole magnetic field is required for the observed optical luminosity.

\section{2. $\gamma$-ray Flux Ratio}

Since outer accelerator models such as the outer gap (e.g., Takata, Wang \& Cheng 2011; Watters \& Romani 2011; Hirotani 2013; Viganò et al. 2015a,b; Viganò \& Torres 2015; Viganò, Torres \& Martín 2015; Pierbattista et al. 2015, 2016) and the current sheet models (e.g., Bai \& Spitkovsky 2010; Kalapotharakos et al. 2012a; Petrova 2013; Kalapotharakos, Harding \& Kazanas 2014; Cerutti. Philippov \& Spitkovsky 2016) could explain the observed $\gamma$-ray emission features, $\gamma \gamma$ scenario is expected to work in $\gamma$-ray pulsars. However, the observed $\gamma$-ray to $\mathrm{X}$-ray flux ratios for a significant fraction of $\gamma$-ray pulsars are much smaller than that in $\gamma \gamma$ scenario (left panel of figure 6). For some low- $L_{\text {sd }}$ $\gamma$-ray pulsars, the observed $\gamma$-ray to optical flux ratios are also smaller than that in $\gamma \gamma$ scenario (right panel of figure 6). In order to explain the observed flux ratios, some additional mechanisms are required. Since the low- $L_{\mathrm{sd}}$ pulsars also show the modulation phenomena in radio (Wang, Manchester \& Johnston 2007), the additional mechanisms may be related to the origins of those phenomena. We consider some possibilities to resolve the flux ratio discrepancies.

In our model, we neglect the thermal emission from entire surface of a neutron star as seed photons in $\gamma \gamma$ pair creation. Using minimum cooling scenario (Page et al. 2004), the effective temperature of the entire surface is $T_{\text {sur }} \gtrsim 10^{6} \mathrm{~K}$ for the pulsars with their age $\tau \lesssim 10^{5}$ yr, which corresponds to the range of spin-down luminosity of $\gamma$-ray pulsars, $L_{\mathrm{sd}} \sim 10^{36} \mathrm{erg} \mathrm{s}^{-1}$. For the age $\tau \sim 10^{5} \mathrm{yr}$, the temperature is $T_{\text {sur }} \sim 10^{6} \mathrm{~K}$ and the surface thermal luminosity is $L_{\text {sur }} \sim 10^{33} \mathrm{erg} \mathrm{s}^{-1}$, which corresponds to $\sim 10^{-3}-10^{-2} L_{\text {sd }}$. Then, the number of the seed photons $N_{\text {sur }} \propto L_{\text {sur }} / T_{\text {sur }}$ is a factor of 3-30 times larger than that in our fiducial case with $L_{\mathrm{pc}} \sim 10^{-3} L_{\mathrm{sd}}$ and $T_{\mathrm{pc}} \sim 10^{6.5} \mathrm{~K}$. The seed photon number is proportional to the optical depth $\tau_{\gamma \gamma}$ (equation 13) and the optical depth is proportional to the synchrotron luminosity $L_{\text {syn }} \propto \tau_{\gamma \gamma}$ (equations 11 and 12). Taking into account the thermal emission from the entire surface, the luminosity $L_{\text {syn }}$ could increase and the luminosity ratio $L_{\text {cur }} / L_{\text {syn }}$ could decrease by a factor of $\sim 3-30$. However, for pulsars with $L_{\mathrm{sd}} \lesssim 10^{35} \mathrm{erg} \mathrm{s}^{-1}$, the thermal photons from the entire surface do not contribute to the optical depth $\tau_{\gamma \gamma}$ (e.g., Yakovlev \& Pethick 2004).

We consider a possible solution that not only the outer accelerator works to emit the observed $\gamma$-ray emission, but also $\mathrm{B} \gamma$ scenario could work to contribute to the observed non-thermal emission to explain the observed X-ray and optical emission for low- $L_{\mathrm{sd}} \gamma$-ray pulsars. Since the strong absorption feature have not been seen in the observed $\gamma$-ray spectra even in low- $L_{\text {sd }} \gamma$-ray pulsars (Abdo et al. 2013), the observed $\gamma$-ray emission comes from the outer magnetosphere where $\gamma \gamma$ scenario works. On the other hand, in $\gamma \gamma$ scenario, the observed lumi- 
nosities in X-ray and optical bands exceed the maximum luminosity, and the observed $\gamma$-ray to X-ray and optical flux ratios $F_{\gamma} / F_{\mathrm{X}}$ and $F_{\gamma} / F_{\mathrm{opt}}$ are much smaller than the ratio $L_{\text {cur }} / L_{\text {syn }}$ for low- $L_{\text {sd }} \gamma$-ray pulsars. In B $\gamma$ scenario, the observed X-ray and optical luminosities are lower than the maximum synchrotron luminosities. In addition, most of $\gamma$-ray photons are absorbed in $\mathrm{B} \gamma$ process, so that the flux ratios $F_{\gamma} / F_{\mathrm{X}}$ and $F_{\gamma} / F_{\text {opt }}$ could be reduced. Then, if the most of the Poynting flux converts to the particle energy at the inner magnetosphere and the remaining Poynting flux converts at the outer magnetosphere, the observed flux ratios should become lower than those in $\gamma \gamma$ scenario. The coexistence of the multiple acceleration regions in the magnetosphere has been suggested by Yuki \& Shibata (2012); Petrova (2013). Recently, such emission models are also suggested to explain the observed light curves of $\gamma$-ray pulsar PSR J1813-1246 in X-ray and $\gamma$-ray (Marelli et al. 2014). For the pulsars with $L_{\mathrm{sd}}<10^{35} \mathrm{erg} \mathrm{s}^{-1}$, the fraction of the population with $\gamma$-ray detection significantly decreases (Laffon et al. 2015). Although the distance, the inclination angle, and the viewing angle affect the $\gamma$ ray detectability, the relative activities of inner and outer accelerators may also affect it. The trend in the $\gamma$-ray detectability reported by Laffon et al. (2015) is consistent with the picture that the energy conversion efficiency $\eta$ at the inner accelerator is larger than that at the outer accelerator for low- $L_{\text {sd }}$ pulsars, while most of Poynting flux converts to the particle energy flux at the outer magnetosphere for high- $L_{\mathrm{sd}}$ pulsars. The multiple emission regions have some constraints such that electromagnetic cascade sites do not reside on the same magnetic field line in the magnetosphere (e.g., Yuki \& Shibata 2012; Kisaka, Asano \& Terasawa 2016). These constraints could be reflected in the shape of observed light curves (e.g., Romani \& Yadigaroglu 1995). . The non-dipole field could also affect the observed light curves. In particular, for the non-thermal X-ray emission from PSR J1741-2041, we should take into account the effect of nondipole magnetic field at the synchrotron emission region (figure 5). Unfortunately, the light curves of non-thermal component for most of low- $L_{\text {sd }}$ pulsars are crude in current observations. We expect that further observations by such as $N u S T A R$ and NICER will clarify the detailed shape of the light curve.

There may be another energy source such as the dissipation of the stellar magnetic field as considered in the magnetar model (e.g., Duncan \& Thompson 1992). In our model, the total energy flux of primary particles is limited by the spin-down luminosity (equation 16). Additional energy sources could make the luminosity exceed the spin-down limit. In fact, magnetar-like bursts and following X-ray flux enhancements are detected from apparently rotation-powered pulsars, PSRs J1846-0258 and J1119-6127 Gavriil et al. 2008; Archibald et al. 2016). For PSR J1119-6127, the enhancement of the power-law component was seen in the X-ray spectrum (Archibald et al. 2016), which emission mechanism may be the synchrotron radiation. Significant fraction of rotation-powered pulsars may also have the dissipative magnetic field, although bursting pulsars have relatively high magnetic field strength $B_{\mathrm{s}} \gtrsim B_{\mathrm{q}}$. Here, we introduce the energy flux $L_{\mathrm{B}}$ via the dissipation of the mag- netic field and its normalized value, $\epsilon_{\mathrm{B}} \equiv L_{\mathrm{B}} / L_{\mathrm{sd}}$. We assume that the available magnetic field energy is comparable to the dipole field, $E_{\mathrm{B}} \sim(4 \pi / 3) R_{\mathrm{ns}}^{3}\left(B_{\mathrm{s}}^{2} / 8 \pi\right)$. Then, the condition for not exhausting the total magnetic energy $E_{\mathrm{B}}$ via the dissipation within their lifetime $\tau \sim \tau_{\mathrm{C}}$ is given by (e.g., Zhang \& Harding 2000b)

$$
\epsilon_{\mathrm{B}}<E_{\mathrm{B}} /\left(L_{\mathrm{sd}} \tau\right)
$$

From equation (47), the steady emission condition in the lifetime is derived as,

$$
\dot{P} \gtrsim 5 \times 10^{-11} \epsilon_{\mathrm{B}} P_{0}^{-3} \mathrm{~s} \mathrm{~s}^{-1} .
$$

Assuming that most of the energy flux of dissipated magnetic field converts to the kinetic energy flux of the primary particle, the energy flux $\epsilon_{\mathrm{B}}$ has to be larger than unity to exceed the spin-down luminosity as an energy source. Only soft $\gamma$-ray repeaters and anomalous X-ray pulsars satisfy this condition (Zhang \& Harding 2000b). Even if we assume that all dissipated energy flux finally converts to the non-thermal luminosity, the parameter $\epsilon_{\mathrm{B}}$ should be an order of $\sim 10^{-3}$ for X-ray band and $\sim 10^{-5}$ for optical band to cover the observed luminosity. We show lines of equation (48) with $\epsilon_{\mathrm{B}}=10^{-3}$ and $10^{-5}$ in the left and right panels of figure 4 as green dotted lines, respectively. In X-ray band, no $\gamma$-ray pulsar satisfies the required condition with $\epsilon_{\mathrm{B}}=10^{-3}$. In optical band, an half of $\gamma$-ray pulsars could satisfy the condition with $\epsilon_{\mathrm{B}}=10^{-5}$. Therefore, if the magnetic field dissipation is the dominant energy source, their non-thermal luminosity does not stay constant in their lifetime unless the dissipation of the non-dipole component dominates the total energy $E_{\mathrm{B}}$. The effects of the additional energy source should also be discussed in a model of magnetar wind nebula (Tanaka 2016).

There may be another mechanism to give the pitch angle to the secondary particles such as a plasma instability (e.g., Machabeli \& Usov 1979; Machabeli et al. 2000) and cycrotron resonant absorption of radio photons (e.g., Lvubarskii \& Petrova 1998; Harding et al. 2008). Then, a part of the momentum of secondary particles parallel to the magnetic field could be converted to the perpendicular component, and is used to emit via synchrotron radiation. This case corresponds to $\gamma \gamma$ scenario with $\alpha \sim 1$. Even in this case and assuming $\gamma_{\mathrm{s} \text {, pair }} \sim \gamma_{\mathrm{s}, \mathrm{syn}}$, from equation (28), the maximum efficiency $\epsilon_{\mathrm{syn}}$ is limited by the number of created secondary particles, $\epsilon_{\text {syn }} \lesssim \tau_{\gamma \gamma}$. For typical $\gamma$-ray pulsars with $\epsilon_{\mathrm{pc}} \sim 10^{-3}, T_{\mathrm{pc}} \sim 10^{6} \mathrm{~K}$ and $P_{0} \sim 0.1 \mathrm{~s}$, the optical depth is $\tau_{\gamma \gamma} \lesssim 10^{-4}$, which is smaller than the observed non-thermal efficiency in the X-ray band.

The synchrotron radiation from pairs created by ingoing curvature photons may also contribute to the observed non-thermal emission. We only consider that the primary particles emit curvature radiation with outgoing direction. For the inwardly emitting curvature photons, the collision angle with thermal X-ray photons could be $\cos \theta \sim-1$. Then, the optical depth $\tau_{\gamma \gamma}$ could be $\sim\left(R_{\mathrm{lc}} / r\right)$ times higher than the outward case. However, using the limitation of the luminosity of the thermal radiation from the heated polar cap, the conversion efficiency should be $\eta \lesssim 10^{-2}$ even if we consider the cooling via curvature radiation for the energy flux of the ingoing primary particles. The 
expected flux of synchrotron radiation from pairs created from curvature photons emitted by ingoing primary particles is comparable to or lower than that from the outgoing pairs (Kisaka \& Tanaka 2014, 2015). Note that in some geometrical conditions, the inward synchrotron emission may dominantly contribute to the observed non-thermal emission (Takata, Chang \& Shibata 2008; Kisaka \& Kojima 2011; Wang. Takata \& Cheng 2013). While the observed non-thermal X-ray luminosity is within our model upper limit, since the inward $\gamma$-ray could be effectively absorbed, the flux ratio $F_{\mathrm{X}} / F_{\gamma}$ could be lower than our model lower limit. The detailed light curve in both $\gamma$-ray and X-ray bands helps to show whether the contribution of the inward emission to the observed non-thermal emission is less significant.

Except for the synchrotron radiation, inverse Compton scattering may work to contribute to the observed non-thermal emission (e.g., Zhang \& Harding 2000a). For the non-resonant Compton scattering, the efficiency should be low because of the small value of the optical depth as seen in $\gamma \gamma$ scenario. In the case of resonant Compton scattering, the resonant condition is

$$
E_{\mathrm{pc}} \gamma_{\mathrm{s}}\left(1-\cos \theta_{\mathrm{col}}\right)=\hbar \frac{e B}{m_{\mathrm{e}} c},
$$

where $\gamma_{\mathrm{s}}$ is the Lorentz factor of the secondary particles.
The energy of the scattered photons is

$$
h \nu_{\mathrm{obs}}=2 \gamma_{\mathrm{s}} m_{\mathrm{e}} c^{2} \frac{B}{B_{\mathrm{q}}} .
$$

Using equations (49) and (50), the emission region in the case of outgoing primary particles is derived as

$$
r_{\mathrm{res}, 6} \sim 12 \nu_{\mathrm{obs}, \mathrm{keV}}^{-1 / 7} T_{\mathrm{pc}, 6.5}^{-1 / 7} B_{\mathrm{s}, 12}^{5 / 14} L_{\mathrm{sd}, 31}^{-1 / 28} .
$$

This is much smaller than the lower limit $r_{\gamma \gamma}$ from the condition of the $\gamma \gamma$ pair creation (equation 39). Only the inner accelerator where $\mathrm{B} \gamma$ process works could supply the scattering particles at the resonant region $r_{\text {res }}$. Then, if the resonant Compton scattering contributes to the observed luminosity for the $\gamma$-ray pulsars, both the inner and outer accelerators work to produce $\gamma$-ray, X-ray and optical emissions. Thus, the inverse Compton model also requires the existence of the multiple particle acceleration sites in the magnetosphere.

We are grateful to the anonymous referee for useful suggestions. We would like to thank K. Asano, Y. Ohira, S. Shibata and J. Takata for fruitful discussions. This work is supported by KAKENHI 16J06773 (S.K.), 24000004 (S.J.T.).

\section{REFERENCES}

Abdo, A. A., Ackermann, M., Atwood, W. B., et al. 2009, ApJ, 696,1084

Abdo, A. A., Ajello, M., Allafort, A., et al. 2013, ApJS, 208, 17 Archibald, R. F., Kaspi, V. M., Tendulkar, S. P., \& Scholz, P. 2016, ApJL, 829, L21

Arons, J., \& Scharlemann, E. T. 1979, ApJ, 231, 854

Backer, D. C. 1970a, Nature, 228, 42

Backer, D. C. 1970b, Nature, 228, 1297

Bai, X.-N., \& Spitkovsky, A. 2010, ApJ, 715, 1282

Becker, W. 2009, in Becker W., ed., Astrophys. Space Sci. Libr. Vol. 357, Neutron Stars and Pulsars. Springer, Berlin

Becker, W., \& Trümper, J. 1997, A\&A, 326, 682

Belyaev, M. A. 2015, MNRAS, 449, 2759

Beronya, D. M., Shibanov, Y. A., Zyuzin, D. A., \& Komarova, V. N. 2015, JPhCS, 661, 012001

Cerutti, B., \& Beloborodov, A. M. 2016, arXiv:1611.04331

Cerutti, B., Philippov, A. A., Parfrey, K., \& Spitkovsky, A. 2015, MNRAS, 448, 606

Cerutti, B., Philippov, A. A., \& Spitkovsky, A. 2016, MNRAS, 457, 2401

Chen, A. Y., \& Beloborodov, A. M. 2014, ApJL, 795, L22

Cheng, K. S., Ho, C., \& Ruderman, M. 1986, ApJ, 300, 522

Daugherty, J. K., \& Harding, A. K. 1982, ApJ, 252, 337

Daugherty, J. K., \& Harding, A. K. 1983, ApJ, 273, 761

Duncan, R. C., \& Thompson, C. 1992, ApJL, 392, L9

Erber, T. 1966, RvMP, 38, 626

Gavriil, F. P., Gonzalez, M. E., Gotthelf, E. V., et al. 2008, Science, 319, 1802

Geppert, U., \& Viganò, D. 2014, MNRAS, 444, 3198

Goldreich, P., \& Julian, W. H. 1969, ApJ, 157, 869

Halpern, J. P., \& Ruderman, M. 1993, ApJ, 415, 286

Harding, A. K. 1981, ApJ, 245, 267

Harding, A. K., \& Lai, D. 2006, RPPh, 69, 2631

Harding, A. K., Stern, J. V., Dyks, J., \& Frackowiak, M. 2008, ApJ, 680, 1378

Hermsen, W., Hessels, J. W. T., Kuiper, L., et al. 2013, Science, 339,436

Hirotani, K. 2013, ApJ, 766, 98

Kalapotharakos, C., Harding, A. K., Kazanas, D., \& Contopoulos, I. 2012a, ApJ, 749, 2

Kalapotharakos, C., Kazanas, D., Harding, A. K., \& Contopoulos, I. 2012b, ApJ, 749, 2
Kalapotharakos, C., Harding, A. K., \& Kazanas, D. 2014, ApJ, 793, 97

Karastergiou, A., \& Johnston, S. 2007, MNRAS, 380, 1678

Kargaltsev, O., Durant, M., Pavlov, G. G., \& Garmire, G. P. 2012, ApJS, 201, 37

Kargaltsev, O., \& Pavlov, G. G. 2008, in AIP Conf. Ser. 983, 40 Years of Pulsars: Millisecond Pulsars, Magnetars and More, ed. C. Bassa, Z. Wang, A. Cumming, \& V. Kaspi (Melville, NY: AIP), 171

Kirichenko, A., Danilenko, A., Shternin, P., et al. 2015, ApJ, 802, 17

Kisaka, S., Asano, K., \& Terasawa, T. 2016, ApJ, 829, 12

Kisaka, S., \& Kojima, Y. 2011, ApJ, 739, 14

Kisaka, S., \& Tanaka, S. J. 2014, MNRAS, 443, 2063

Kisaka, S., \& Tanaka, S. J. 2015, Proceedings of the 5th Fermi Symposium, arXiv:1503.00483

Kramer, M., Lyne, A. G., O’Brien, J. T., Jordan, C. A., \& Lorimer, D. R. 2006, Science, 312, 549

Laffon, H., Smith, D. A., Guillemot, L., for the Fermi-LAT Collaboration 2015, Proceedings of the 5th Fermi Symposium, arXiv: 1502.03251

Li, J., Spitkovsky, A., \& Tchekhovskoy, A. 2012, ApJ, 746, 60

Lyne, A., Hobbs, G., Kramer, M., Stairs, I., \& Stappers, B. 2010, Science, 329, 408

Lyubaskii, Y. E. 1996, A\&A, 311, 172

Lyubaskii, Y. E., \& Petrova, S. A. 1998, A\&A, 337, 433

Machabeli, G. Z., Luo, Q., Melrose, D. B., \& Vladimirov, S. 2000, MNRAS, 312, 51

Machabeli, G. Z., \& Usov, V. V. 1979, SvAL, 5, 238

Manchester, R. N., Hobbs, G. B., Teoh, A., \& Hobbs, M. 2005, AJ, 129, 1993

Marelli, M., Harding, A., Pizzocaro, D., et al. 2014, ApJ, 795, 168

Marelli, M., Mignani, R. P., De Luca, A., et al. 2015, ApJ, 802, 78

Mereghetti, S., Kuiper, L., Tiengo, A., et al. 2016, ApJ, 831, 21

Mereghetti, S., Tiengo, A., Esposito, P., \& Turolla, R. 2013, MNRAS, 435, 2568

Mignani, R. P., Pavlov, G. G., \& Kargaltsev, O. 2008, A\&A, 488, 1027

Mignani, R. P., Pavlov, G. G., \& Kargaltsev, O. 2011, A\&A, 531, A105

Mignani, R. P., Rea, N., Testa, V., et al. 2016a, MNRAS, 461, 4317 
Mignani, R. P., Testa, V., Marelli, M., et al. 2016b, MNRAS, 463, 2932

Mignani, R. P., Testa, V., Marelli, M., et al. 2016c, ApJ, 825, 151 O'Dell, S. L., \& Sartori, L. 1970, ApJL, 161, L63

Page, D., Lattimer, J. M., Prakash, M., \& Steiner, A. W. 2004, ApJS, 155,623

Pétri, J. 2012, MNRAS, 424, 2023

Petrova, S. A. 2013, ApJ, 764, 129

Philippov, A. A., Cerutti, B., Tchekhovskoy, A., \& Spitkovsky, A. 2015, ApJL, 815, L19

Philippov, A. A., \& Spitkovsky, A. 2014, ApJL, 785, L33

Philippov, A. A., Spitkovsky, A., \& Cerutti, B. 2015, ApJL, 801, L19

Pierbattista, M., Harding, A. K., Grenier, I. A., et al. 2015, A\&A, 575, A3

Pierbattista, M., Harding, A. K., Gonthier, \& P. L., Grenier, I. A. 2016, A\&A, 588, A137

Pilia, M., Hessels, J. W. T., Stappers, B. W., et al. 2016, A\&A, 586, A92

Posselt, B., Pavlov, G. G., Manchester, R. N., Kargaltsev, O., \& Garmire, G. P. 2012a, ApJ, 749, 146

Posselt, B., Arumugasamy, P., Pavlov, G. G., et al. 2012b, ApJ, 761,117

Possenti, A., Cerutti, R., Colpi, M., \& Mereghetti, S. 2002, A\&A, 387, 993

Prinz, T., \& Becker, W. 2015, arXiv:1511.07713

Romani, R. W. 1996, ApJ, 470, 469

Romani, R. W., \& Yadigaroglu, I.-A. 1995, ApJ, 438, 314

Rudak, B., \& Dyks, J. 1999, MMNRAS, 303, 477

Ruderman, M. A., \& Sutherland, P. G. 1975, ApJ, 196, 51

Seward, F. D., \& Wang, Z.-R. 1988, ApJ, 332, 199

Shibanov, Y., Danilenko, A., Zharikov, S., Shternin, P., \& Zyuzin, D. 2016, ApJ, 831, 112

Shibata, S., Watanabe, E., Yatsu, Y., Enoto, T., \& Bamba, A. 2016, ApJ, 833, 59
Sturrock, P. A. 1971, ApJ, 164, 529

Szary, A., Gil, J., Zhang, B., Haberl, F., Melikidze, G.-I. Geppert, U., Mitra, D., \& Xu, R.-X. 2017, ApJ, 835, 178 Szary, A., Melikidze, G.-I., \& Gil, J. 2015, MNRAS, 447, 2295

Takata, J., \& Chang, H.-K. 2009, MNRAS, 392, 400

Takata, J., Chang, H.-K., \& Shibata, S. 2008, MNRAS, 386, 748

Takata, J., Wang, Y., \& Cheng, K. S. 2011, MNARS, 415, 1827

Tanaka, S. J. 2016, ApJ, 827, 135

Tanaka, S. J., \& Takahara, F. 2010, ApJ, 715, 1248

Tanaka, S. J., \& Takahara, F. 2011, ApJ, 741, 40

Tanaka, S. J., \& Takahara, F. 2013, MNRAS, 429, 2945

Tang, A. P. S., Takata, J., Jia, J. J., \& Cheng, K. S. 2008, ApJ, 676,562

Timokhin, A. N., \& Harding, A. K. 2015, ApJ, 810, 144

Viganò, D., \& Torres, D. F. 2015, MNRAS, 449, 3755

Viganò, D., Torres, D. F., Hirotani, K., \& Pessah, M. E. 2015a, MNRAS, 447, 2631

Viganò, D., Torres, D. F., Hirotani, K., \& Pessah, M. E. 2015b, MNRAS, 447, 2649

Viganò, D., Torres, D. F., \& Martín, J. 2015, MNRAS, 453, 2599

Wang, N., Manchester, R. N., \& Johnston, S. 2007, MNRAS, 377, 1383

Wang, R.-B., \& Hirotani, K. 2011, ApJ, 736, 127

Wang, Y., Takata, J., \& Cheng, K. S. 2013, ApJ, 764, 51

Watters, K. P., \& Romani, R. W. 2011, ApJ, 727, 123

Weltevrede, P., \& Johnston, S. 2008, MNRAS, 391, 1210

Yakovlev, D. G., \& Pethick, C. J. 2004, ARA\&A, 42, 169

Yuki, S., \& Shibata, S. 2012, PASJ, 64, 43

Zavlin, V. E., \& Pavlov, G. G. 2004, ApJ, 616, 452

Zhang, L., \& Cheng, K. S. 1997, ApJ, 487, 370

Zhang, B., \& Harding, A. K. 2000a, ApJ, 532, 1150

Zhang, B., \& Harding, A. K. 2000b, ApJL, 535, L51

Zharikov, S., \& Mignani, R. P. 2013, MNRAS, 435, 2227

Zharikov, S., Shibanov, Y., \& Komarova, V. 2006, AdSpR, 37, 1979 